\title{
Id genes are essential for early heart formation
}

\author{
Thomas J. Cunningham, ${ }^{1,10}$ Michael S. Yu, ${ }^{1,2,10}$ Wesley L. McKeithan, ${ }^{1,3,4,10}$ Sean Spiering, ${ }^{1}$ \\ Florent Carrette, ${ }^{1}$ Chun-Teng Huang, ${ }^{1}$ Paul J. Bushway, ${ }^{2}$ Matthew Tierney, ${ }^{1}$ Sonia Albini, ${ }^{1}$ \\ Mauro Giacca, ${ }^{5}$ Miguel Mano, ${ }^{6}$ Pier Lorenzo Puri, ${ }^{1,7}$ Alessandra Sacco, ${ }^{1}$ Pilar Ruiz-Lozano, ${ }^{1,8}$ \\ Jean-Francois Riou, ${ }^{9}$ Muriel Umbhauer, ${ }^{9}$ Gregg Duester, ${ }^{1}$ Mark Mercola, ${ }^{1,4,11}$ and Alexandre R. Colas ${ }^{1,11}$ \\ ${ }^{1}$ Sanford Burnham Prebys Medical Discovery Institute, La Jolla, California, 92037, USA; ${ }^{2}$ Department of Bioengineering, \\ University of California at San Diego, La Jolla, California 92037, USA ${ }^{3}$ Graduate School of Biomedical Sciences, Sanford Burnham \\ Prebys Medical Discovery Institute, La Jolla, California 92037, USA; ${ }^{4}$ Department of Medicine and Cardiovascular Institute, \\ Stanford University, Palo Alto, California 94305, USA; ${ }^{5}$ International Centre for Genetic Engineering and Biotechnology, 34149 \\ Trieste, Italy; ${ }^{6}$ Center for Neuroscience and Cell Biology (CNC), University of Coimbra, 3004-504 Coimbra, Portugal; ${ }^{7}$ Istituti di \\ Ricovero e Cura a Carattere Scientifico, Fondazione Santa Lucia, 00179 Rome, Italy; ${ }^{8}$ Regencor, Inc., Los Altos, California 94022 , \\ USA; ${ }^{9}$ UMR 7622 Developmental Biology, Sorbonne Universités, University Pierre and Marie Curie, F- 75005 Paris, France
}

Deciphering the fundamental mechanisms controlling cardiac specification is critical for our understanding of how heart formation is initiated during embryonic development and for applying stem cell biology to regenerative medicine and disease modeling. Using systematic and unbiased functional screening approaches, we discovered that the Id family of helix-loop-helix proteins is both necessary and sufficient to direct cardiac mesoderm formation in frog embryos and human embryonic stem cells. Mechanistically, Id proteins specify cardiac cell fate by repressing two inhibitors of cardiogenic mesoderm formation-Tcf3 and Foxa2-and activating inducers Evx1, Grrp1, and Mesp1. Most importantly, CRISPR/Cas9-mediated ablation of the entire Id (Id1-4) family in mouse embryos leads to failure of anterior cardiac progenitor specification and the development of heartless embryos. Thus, Id proteins play a central and evolutionarily conserved role during heart formation and provide a novel means to efficiently produce cardiovascular progenitors for regenerative medicine and drug discovery applications.

[Keywords: cardiac progenitors; cardiac mesoderm specification; heartless; Id proteins; CRISPR/Cas9-mediated quadruple knockout; platform for cardiac disease modeling and drug discovery]

Supplemental material is available for this article.

Received April 12, 2017; revised version accepted July 17, 2017.

Heart formation begins during gastrulation with the specification of cardiogenic mesoderm progenitors (CMPs) that migrate anteriorly to form the cardiac primordium that assembles into the fully formed heart (Buckingham et al. 2005; Kelly et al. 2014; Meilhac et al. 2015). Intense research over the past two decades has led to the identification of extracellular signals that initiate cardiogenesis (Schultheiss et al. 1997; Marvin et al. 2001; Schneider and Mercola 2001; Pandur et al. 2002; Collop et al. 2006; Kattman et al. 2006; Foley et al. 2007; Laflamme et al. 2007; Yang et al. 2008; Lian et al. 2013). In contrast, current knowledge of the intracellular mediators controlling this process is very fragmentary. Discovering such factors would have major implications (1) for appreciating how cardiogenesis is normally initiated, as embryos lacking

\footnotetext{
${ }^{10}$ These authors contributed equally to this work.

${ }^{11}$ These authors contributed equally to this work. Corresponding author: acolas@sbpdiscovery.org

Article published online ahead of print. Article and publication date are online at http://www.genesdev.org/cgi/doi/10.1101/gad.300400.117. Freely available online through the Genes \& Development Open Access option.
}

cardiac progenitors fail to form a heart (Zhao et al. 2008), and (2) for informing the development of regenerative and disease modeling technologies (Mercola et al. 2013; Moretti et al. 2013).

Basic helix-loop-helix (bHLH) transcription factors Mesp1 and Mesp2 (Saga et al. 2000) under the control of T-box factor Eomes (Costello et al. 2011) regulate at least part of this process in mesoderm cells by directing the expression of genes involved in cardiac specification (Hand2, Gata4, Nkx2.5, and Myocd) and cellular migration (Prickle1 and RasGRP3) while actively repressing genes regulating pluripotency (Oct4, Nanog, and Sox2) and early mesoderm $(T)$ and endoderm (Foxa2 and Sox17) fates (Bondue et al. 2008; Costello et al. 2011; Chiapparo et al. 2016). Although these observations suggest that Mesp1/2 genes could act as master regulators of multipotent cardiovascular specification, retrospective lineage analysis (Saga et al. 2000; Yoshida et al. 2008) and in vitro differentiation studies (Chan et al. 2013) have

(C) 2017 Cunningham et al. This article, published in Genes \& Development, is available under a Creative Commons License (Attribution $4.0 \mathrm{In}$ ternational), as described at http://creativecommons.org/licenses/by/4.0/. 
shown that Mesp1-expressing cells also contribute to a wide range of noncardiac derivatives, including hematopoietic precursors, skeletal muscle cells, and head mesenchyme. Therefore, additional effectors responsible for specifying cardiac cell fate remain to be discovered.

We reported recently that attenuating Acvr1b signaling in mesendoderm segregates cardiogenic mesoderm from endoderm, whereas persistent Acvrlb signaling drives cells to form endoderm (Colas et al. 2012). Thus, we hypothesized that genes induced in response to Acvr1b signaling inhibition might be key determinants of cardiogenic mesoderm formation. We took a systematic and unbiased approach to functionally test the necessity and sufficiency of the genes modulated by Acvrlb signaling blockade. Unexpectedly, we first identified Id1, a HLH transcriptional regulator, as a single factor sufficient to control the emergence of $\mathrm{Kdr}^{+}$CMPs both in mouse and human embryonic stem cells (mESCs and hESCs, respectively). Mechanistically, we discovered that Id proteins mediate their evolutionarily conserved role by activating the expression of agonists of cardiogenic mesoderm formation (Evx1, Grrp1, and Mesp1) while inhibiting antagonists' activity (Tcf3 and Foxa2). Finally, CRISPR/Cas9mediated deletion of all four Id family members in mouse embryos blocked early cardiac progenitor formation and yielded embryos without a heart. The heartless phenotype was unique to the quadruple knockout, indicating compensatory or redundant functions of the Id proteins in the formation of the cardiac mesoderm. These findings reveal an unexpected role for Id proteins as the earliest determinants of cardiac cell fate in vertebrates.

\section{Results}

\section{Identification of new agonists of cardiogenic mesoderm formation}

mESCs form mesendodermal progenitors $\left(\mathrm{Gsc}^{+}, \mathrm{Foxa}^{+}\right.$, and $\mathrm{T}^{+}$) at days 3-4 of differentiation in response to Activin/Nodal signaling and subsequently differentiate into either Foxa2 $2^{+}$definitive endoderm or $\mathrm{Kdr}^{+}$cardiogenic mesoderm (diagrammed in Fig. 1A; described previously in Colas et al. 2012). Attenuation of Acvr1b drives mesendodermal progenitors to form CMPs marked by Mesp1, Kdr, Cdh11, and Snail expression at days 5-6 rather than endoderm-a process robustly elicited by transfecting mesendodermal progenitors at day 3 with either let7 or miR-18 mimics or siRNAs directed against their respective mRNA targets: Acvr1b or Smad2 (day 3) (Fig. 1A-C; Supplemental Movies S1-2; Colas et al. 2012; McKeithan et al. 2012).

To identify the downstream effectors of cardiogenic mesoderm formation, we analyzed mRNA expression $24 \mathrm{~h}$ after Acvr1b siRNA (siAcvr1b) transfection (day 4). Microarray data revealed 33 genes that were up-regulated (Fig. 1D; Supplemental Table S1) in response to Acvr1b siRNA relative to a scrambled sequence siRNA control, of which 14 were confirmed by quantitative PCR (qPCR) (Fig. 1E). Consistent with a potential role as cell fate regulators, eight of the candidate genes are known regulators of gene transcription, including transcription factors (Evx1, Gbx2, Irx3, Irx5, and Sox9), inhibitors of bHLH transcription factors (Id1 and Id3), and a mediator of DNA demethylation (Gadd45g). Of the six remaining candidates, three are signaling pathway modulators $(F g f b p 3$, Crabp2, and Cxc112), two are involved in RNA processing (Elavl3 and Tnrc6a), and one encodes a protein with two centrosome-associated domains but no known function (Grrp1). Interestingly, none of the 14 candidates were shown previously to directly control cardiogenic mesoderm formation, suggesting that we identified a novel molecular signature marking differentiating CMPs.

Next, we assessed whether siRNA against each of the 14 candidates would block cardiogenic mesoderm formation induced by siAcvrlb using a $K d r$-eGFP reporter system (Colas et al. 2012). Of all of the up-regulated genes, only siRNAs against Grrp1, Evx1, and Id1 significantly decreased the number of $\mathrm{Kdr}^{+}$-expressing cells (Fig. 1F-J) and blunted the induction of cardiogenic mesoderm marker genes, including $K d r$, Mesp1, Snai1, and Cdh11 (Fig. 1K). Thus, Grrp1, Evx1, and Id1 are needed for normal cardiogenic mesoderm differentiation in mESCs.

Spatiotemporal expression of Id1, Grrp1, and Evx1 is consistent with involvement in cardiogenic mesoderm formation

In siAcvr1b conditions, maximal Id 1 expression occurs at day 4 of mESC differentiation, preceding the peaks of Grrp1, Evx1, and Mesp1 expression (Fig. 1L-O). In mice, Id1 is expressed throughout the epiblast in the most proximal region of gastrula stage embryos (embryonic days 6.5-7.25 [E6.5-E7.25]) and the anterior lateral mesoderm where specified cardiac precursors are located (Fig. 1P,T, $\mathrm{T}^{\prime}$; Devine et al. 2014). Id1 transcripts are notably absent from the primitive streak, posterior mesoderm, and definitive endoderm. Grrp1 transcripts are expressed throughout the primitive streak of the embryo (Fig. 1Q,U, $\mathrm{U}^{\prime}$ ). Transverse sections reveal that Grrp1 mRNA is mostly localized in the gastrulating epiblast and rapidly declines as cells migrate away from the primitive streak. Like Id1, Evx1 is expressed in the most proximal region of the embryo within the primitive streak and migrating mesoderm (Fig. 1R, V, $\mathrm{V}^{\prime}$ ). Mesp1 expression marks the early differentiating multipotent mesoderm cells as they emerge from the primitive streak and start to migrate (Fig. 1S,W, W'). Thus, the spatiotemporal expression of candidate transcripts is consistent with their potential involvement in cardiogenic specification in mesendoderm progenitors that normally takes place between E6.5 and E7.5 (Bardot et al. 2017). The data also suggest that $I d 1$ in the gastrulating epiblast may function upstream of Grrp1 and Evx1 to ultimately direct Mesp1 expression in cells that exit the primitive streak (Fig. 1X).

\section{Id1 is sufficient to direct $\mathrm{Kdr}^{+}$cardiogenic mesoderm formation in mESCs and hESCs}

To evaluate whether candidate genes alone or in combination are sufficient to promote cardiogenic mesoderm 
A

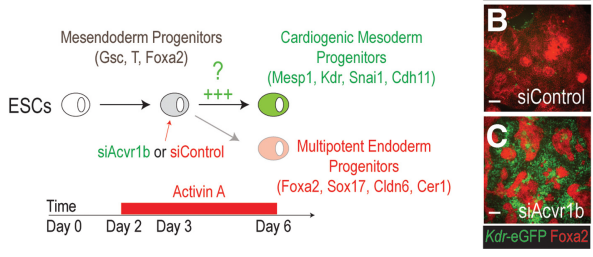

$\begin{array}{lll}\mathbf{F} & 2.07 \quad \text { Day } 6\end{array}$

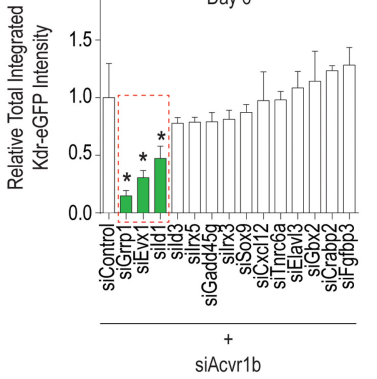

D

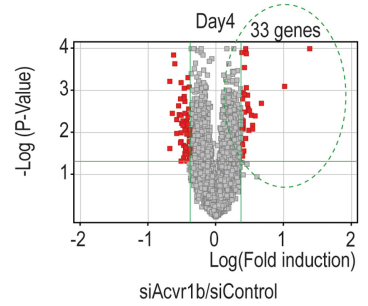

siAcvr1b/siControl

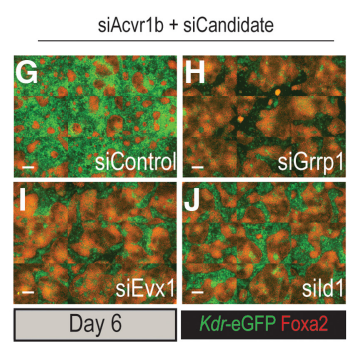

E
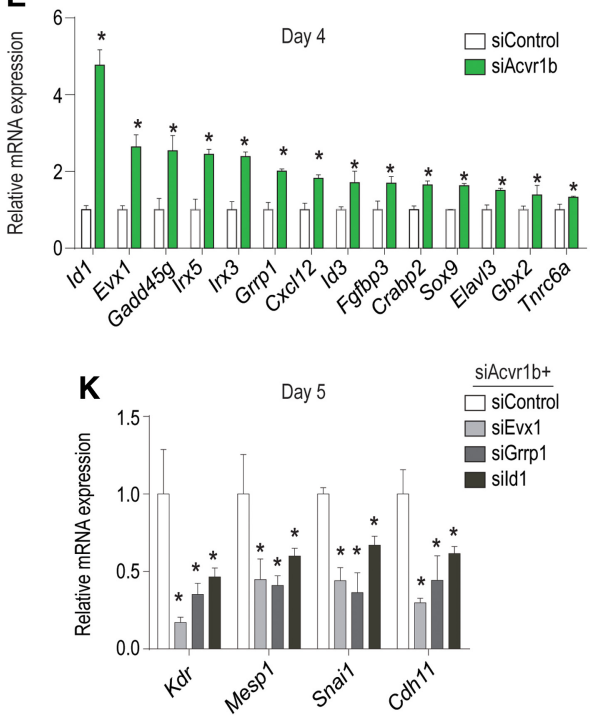

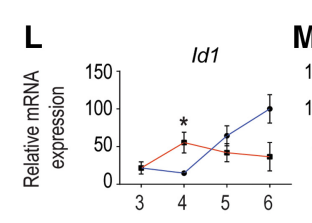

$\mathbf{N}$
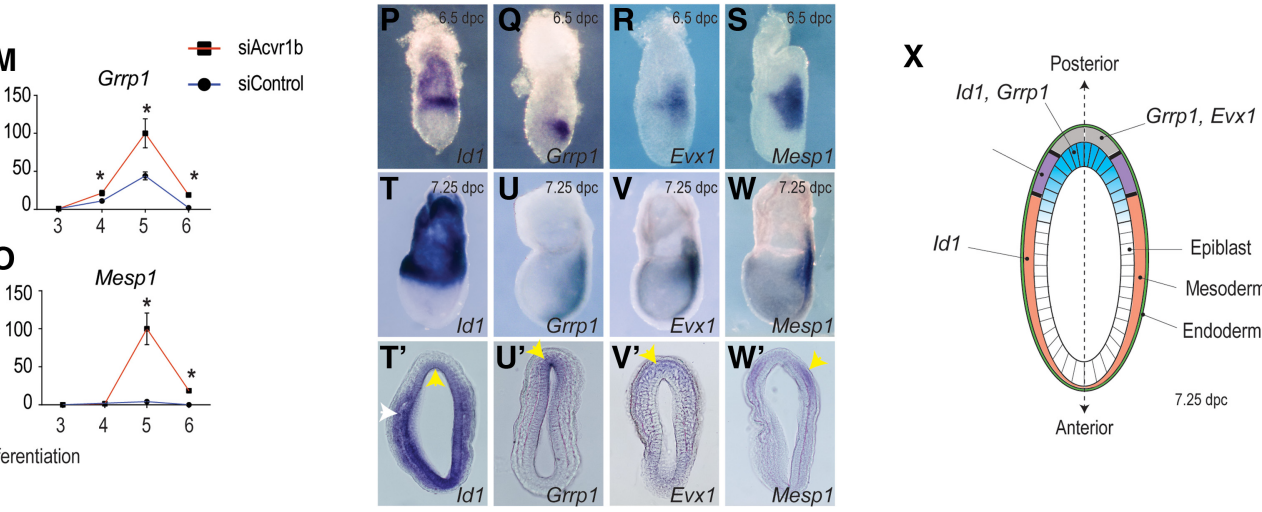

Figure 1. Positive regulators of CMP formation. $(A)$ Schematic of screening strategy to identify new regulators of cardiogenic mesoderm differentiation. $(B, C)$ Immunostaining of $K d r$-eGFP (cardiogenic mesoderm) and Alexa fuor568-Foxa2 (endoderm) showing increased mesoderm differentiation in response to siAcvrlb as compared with siControl at day 6 of differentiation. Bar, $50 \mu \mathrm{m}$. $(D)$ Microarray data reveal that 33 transcripts are up-regulated $(P<0.05)$ at day 4 in response to siAcvrlb as compared with siControl $24 \mathrm{~h}$ after transfection. $(E)$ Quantitative RT-PCR (qRT-PCR) confirmation of the microarray results in $D$, showing that 14 genes are robustly up-regulated in response to siAcvrlb as compared with siControl. $(F)$ siRNA screen of the 14 candidates from $E$ to evaluate their requirement for cardiogenic mesoderm formation induced by siAcvrlb. Differentiation was quantified by induction of $K d r$-eGFP reporter (total integrated intensity) (see the Materials and Methods for details). siGrrp1, siEvx1, and siId1 strongly repressed siAcvrlb-induced cardiogenic mesoderm. (G-T) Representative images of $K d r$-eGFP and Alexa fluor 568-Foxa2 illustrating results presented in $F$. Bar, $50 \mu \mathrm{m}$. $(K)$ qRT-PCR results showing that siGrrp1, siEvx 1, and siId1 markedly repress cardiogenic mesoderm-specific marker (Kdr, Mesp1, Snai1, and Cdh11) expression. (L-O) Temporal expression profiles of Id1, Grrp1, Evx1, and Mesp1 in response to siAcvrlb or siControl from day 3 to day 6 of differentiation. $\left(P-W^{\prime}\right)$ Endogenous expression of Id1, Grrp1, Evx1, and Mesp1 in embryonic day 6.5 (E6.5) and E7.25 mouse embryos by in situ hybridization. (P$W$ ) Whole-mount view. Transverse histological section of the proximal region of $\mathrm{E} 7$ embryos indicating Id1 $\left(T^{\prime}\right)$ expression in the gastrulating epiblast (yellow arrow) and migrating mesoderm (white arrow), Grrp1 ( $\left.U^{\prime}\right)$ expression in the gastrulating epiblast (yellow arrow), and Evx1 $\left(V^{\prime}\right)$ and Mesp1 $\left(W^{\prime}\right)$ expression in the primitive streak (yellow arrow). (X) Schematic representation of an E7.25 embryo transverse section illustrating the different domains of expression of the three candidates. The gastrulating epiblast (blue) indicates the domain where Id1 and Grrp1 expression overlaps. In the primitive streak region (gray), high levels of Evx1 expression are observed with decreased Grrp1 expression. As cells exit the primitive streak and migrate laterally (purple), they start to express Mesp1 along with Evx1. As mesoderm cells migrate more anteriorly (orange), they resume Id1 expression. All qRT-PCR data were normalized to $\beta$-actin mRNA levels. Quantitative data are presented as means $\pm \mathrm{SD}$. $\left.{ }^{*}\right) P<0.05$. All experiments were performed at least in biological quadruplicates.

differentiation, we first generated mESC lines overexpressing all seven possible combinations of the three candidates (Fig. 2A; Supplemental Fig. S1A). The cell lines were treated with Activin A (but not with Acvr1b siRNA), and the resulting differentiation was assessed on day 6. Id1 was sufficient to massively direct ESCs to differentiate toward the $\mathrm{Kdr}^{+}$mesoderm without attenuating Acvrlb expression ( 22-fold over parental mESCs), while the other genes had less potent effects (Fig. 2B-D; Supplemental Fig. S1B-G). Quantitatively, the conversion rate of 

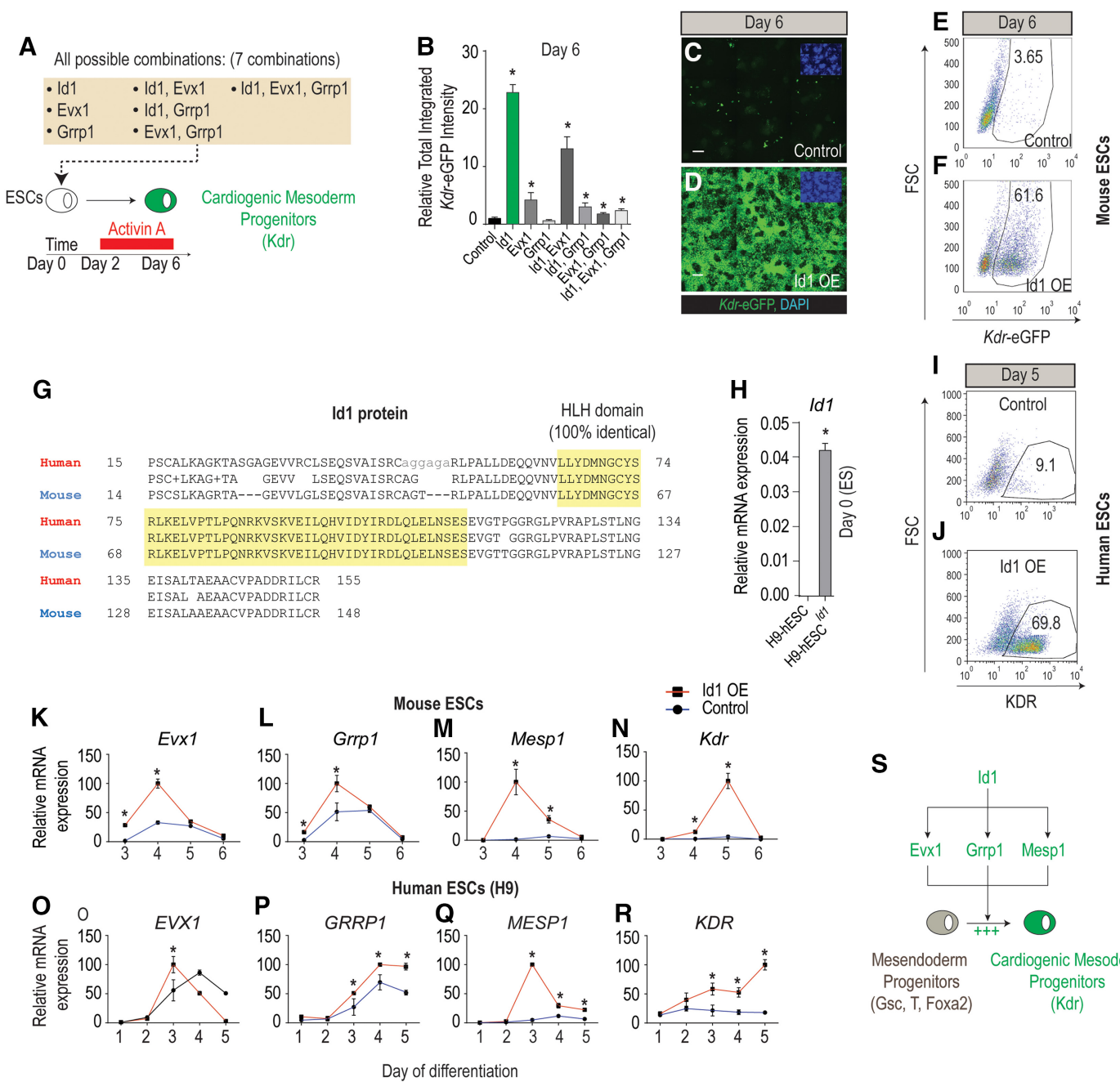

$\mathbf{S}$

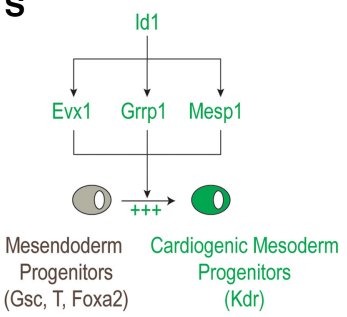

Figure 2. Idl is sufficient to direct $\mathrm{Kdr}^{+}$mesoderm formation in mESCs and hESCs. (A) Schematic of the strategy to evaluate the sufficiency (gain of function) of any of three candidates alone or in combination to promote mesoderm differentiation. (B) Kdr-eGFP fluorescence measurement at day 6 of differentiation in mESCs overexpressing all possible combinations of the three candidates plotted relative to uninfected control levels. $(C, D)$ Representative images of $K d r$-eGFP for Idl-overexpressing versus control mESCs illustrating the results presented in $B$. Bar, $50 \mu \mathrm{m}$. $(E, F)$ Flow cytometry analysis reveals that $61.6 \%$ of $I d 1$-overexpressing mESCs differentiate into $K d r$-eGFP ${ }^{+}$ mesoderm as compared with 3.65\% for control cells at day 6. (G) Alignment and comparison of the mouse (NP_034625.1) Id1 HLH domain and the human (NP_851998.1) Id1 HLH domain using the Protein Blast tool (https://blast.ncbi.nlm.nih.gov) reveals that the amino acid sequence is $100 \%$ identical. $(H)$ qRT-PCR analysis for expression of Id1 in control h9 hESCs versus h9 hESCs stably overexpressing Id 1 measured at day 0 of differentiation. $(I, J)$ Flow cytometry analysis reveals that $69.8 \%$ of Id1-overexpressing h9 hESCs differentiate into $\mathrm{KDR}^{+}$mesoderm at day 5 of differentiation as compared with $9.1 \%$ for control h9 hESCs. $(K-N)$ Temporal mRNA expression profile of procardiogenic mesoderm genes (EVX1 [K], Grrp1 [L], Mesp1 [M], and Kdr [N]) in mESC lines overexpressing Id1 compared with control mESC lines illustrating that Evx1, Grrp1, and Mesp1 mRNA expression peaks at day 4 of differentiation, while $K d r$ mRNA expression peaks at day 5 of differentiation. $(O-R)$ Temporal mRNA expression profiles of $E V X 1(O), G R R P 1(P), M E S P 1(Q)$, and KDR $(R)$ in h9 hESCs stably overexpressing Id1 compared with control h9 hESCs. (S) Model summarizing the procardiogenic role of Id1 by up-regulating the expression of Evx1, Grrp1, and Mesp1 in bipotent mesendoderm progenitors. Quantitative data are presented as means \pm SD. All experiments were performed at least in biological quadruplicates. The insets in the top right corners of all immunostaining images show corresponding DAPI staining.

Id1-overexpressing mESCs into $K d r$-eGFP ${ }^{+}$mesoderm was $\sim 60 \%$ as compared with only $3.65 \%$ for control ESCs (Fig. 2E,F).

Next, we asked whether Id1 functions similarly in hESCs by generating a WiCell (H9) hESC line that stably overexpresses mouse Id1, since the HLH domains are identical across these species (Fig. 2G,H). Consistent with the result with mESCs, Id1 greatly increased the incidence of $\mathrm{KDR}^{+}$mesoderm in Activin A-treated cultures at day 5 from $9.1 \%$ in parental hESCs to $69.8 \%$ in hESC $^{\text {Id } 1}$ (Fig. 2I,J).

Remarkably, the formation of Id1-induced $\mathrm{Kdr}^{+} / \mathrm{KDR}^{+}$ mesoderm progenitors (iMPs) was consistently preceded by the up-regulation of Evx1/EVX1 and Grrp1/GRRP1 
(days $3 / 4$ in mESCs [Fig. 2K,L] and day 3 in hESCs [Fig. 2O, P]) followed by dramatic Mesp1/MESP1 up-regulation ( 67 -fold in mESCs at day 4 and $\sim 20$-fold in hESCs at day 3) (Fig. 2M,Q) and subsequent $K d r / K D R$ up-regulation at day 4 and day 5 , respectively (Fig. 2N,R). Together, these data show that Idl initiates an evolutionarily conserved gene regulatory network (Evx1/EVX1, Grrp1) GRRP1, and Mesp1/MESP1) controlling the formation of $\mathrm{Kdr}^{+} / \mathrm{KDR}^{+}$mesoderm (Fig. 2S).

Next, we asked whether iMPs are bona fide CMPs and thus are able to differentiate into multiple cardiac lineages, including functional cardiomyocytes. To address this question, iMPs were first produced in bulk until day 6 of differentiation for mice or day 5 for humans. At this point, iMPs could be cryopreserved or used fresh. Spontaneous differentiation potential under basal medium conditions without cytokines (Fig. 3A) was assessed by RT-qPCR (Fig. 3B,C) and immunostaining (Fig. 3D; Supplemental Fig. S2A,B) at day 15 of differentiation. The results showed that iMPs spontaneously differentiate into at least four distinct cellular lineages normally present in the heart, including cardiomyocytes (Myh6,
Tnnt2, and Actc1), vascular endothelial cells (Pecam1 and Cdh5), smooth muscle (Myh11), and fibroblasts (Postn and Tagln). Although iMPs are multipotent progenitors, the vast majority of the cells $(\sim 70 \%)$ spontaneously differentiated into $\mathrm{ACTCl}^{+}$cardiomyocytes in hESCs (Fig. 3E). Next, we assessed whether the resulting $\mathrm{ACTCl}^{+}$cells show characteristics of functional cardiomyocytes, which include the rhythmic contractions, intracellular calcium oscillations and action potentials, and response to hormonal stimuli (Burridge et al. 2014; Birket et al. 2015). High-speed optical recording (100 frames per second) (Fig. 3F) revealed that day 15 iMPs contracted rhythmically (Supplemental Movie S3), displayed periodic calcium transients (Fig. 3G, $\mathrm{H}_{\text {; }}$ Supplemental Movie S4) and action potentials (Fig. 3I; Supplemental Movie S5), and showed increased beat rate in response to the $\beta$-adrenergic agonist isoproterenol (Fig. 3J,K; Supplemental Movie S6). In summary, these observations demonstrate that iMPs represent a novel population of bona fide CMPs with a remarkable ability to spontaneously differentiate into functional cardiomyocytes.
A
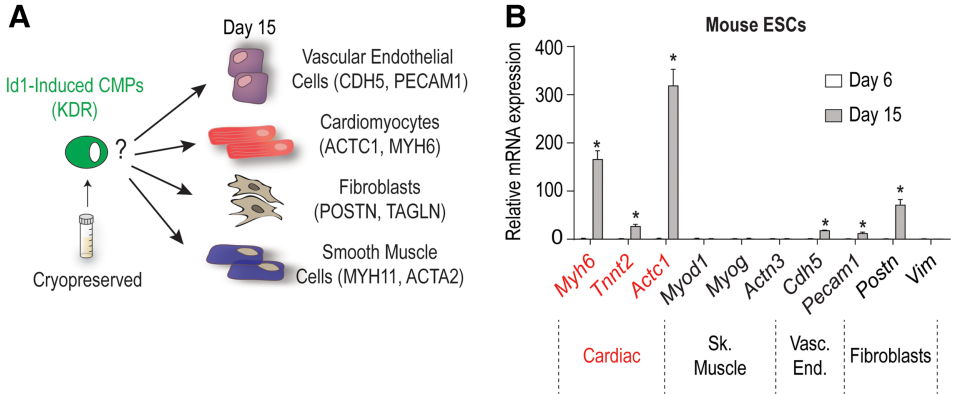

C

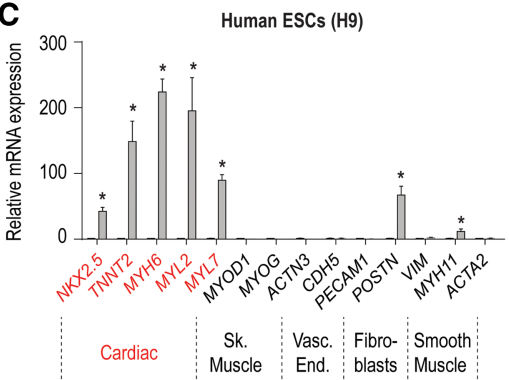

$\mathbf{F}$
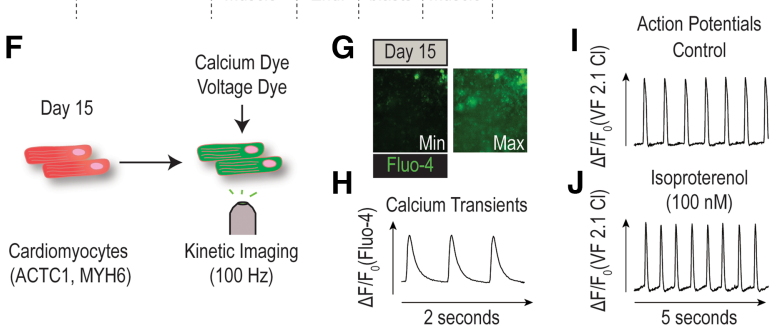

Figure 3. Idl-induced $\mathrm{Kdr}^{+}$mesoderm is cardiogenic. $(A)$ Schematic depicting the prospective differentiation potential of cryopreserved Id1-induced CMPs to multiple cardiovascular cell types. (B) mRNA expression profiling for the spontaneous differentiation potential of mESCs stably overexpressing Id1 to cardiac (Myh6, Tnnt2, and Actc1), skeletal muscle (Myod1, Myog, and Actn3), vascular endothelial (Cdh5 and Pecam1), and fibroblast (Postn and Vim) markers at days 6 and 15 of differentiation. $(C)$ mRNA expression profiling for the spontaneous differentiation potential of h9 hESCs stably overexpressing Id1 to cardiac (NKX2.5, TNNT2, MYH6, MYL2, and $M Y L 7)$, skeletal muscle (MYOD1, MYOG, and ACTN3), vascular endothelial (CDH5 and PECAM1), smooth muscle (MYH11 and ACTA2), and fibroblast (POSTN and VIM) markers at days 5 and 15 of differentiation. (D) Representative immunofluorescence image of cardiomyocytes (ACTC1), vascular endothelial cells (CDH5), and fibroblasts (TAGLN) at day 15 of differentiation in h9 hESCs stably overexpressing Id1. Bar, $50 \mu \mathrm{m}$. (E) Diagram showing quantification of the percentage of $\mathrm{ACTCl}^{+}$(cardiomyocytes), $\mathrm{TAGLN}^{+}$(fibroblasts), and $\mathrm{CDH}^{+}$(vascular endothelial cells) at day 15 of differentiation in h9 hESCs stably overexpressing Id1. (F) Schematic of the work flow for the physiological assessment of cardiomyocytes derived from Id1-overexpressing h9 hESCs using the calcium-sensitive (Fluo-4) and voltage-sensitive (VF2.1 Cl) (Miller et al. 2012) dyes. (G) Representative images illustrating the minimum and maximum changes in fluorescence of Fluo-4 in cardiomyocytes derived from Id1-overexpressing h9 hESCs. $(H)$ Representative calcium transient trace of day 15 cardiomyocytes derived from Id1-overexpressing h9 hESCs. $(I, J)$ Representative action potential traces of cardiomyocytes derived from Id1-overexpressing h9 hESCs in control conditions $(I)$ or in response to isoproterenol $(J)$ measured optically with VF2.1 Cl. $(K)$ Beat rate quantification of cardiomyocytes derived from Id1-overexpressing h9 hESCs indicating an increase in beating frequency in response to $100 \mathrm{nM}$ isoproterenol treatment as compared with vehicle and measured with VF2.1 Cl. Quantitative data are presented as means \pm SD. All experiments were performed at least in biological quadruplicates. The insets in the top right corners of all immunostaining images shows corresponding DAPI staining. 


\section{Id1 promotes cardiogenic mesoderm differentiation by inhibiting Tcf3 and Foxa2}

Id proteins do not directly bind DNA but regulate transcription by antagonizing the function of bHLH transcription factors through their HLH domains (Kee 2009). Their canonical partners are class I bHLH transcription factors (E proteins) Tcf3, Tcf4, and Tcf12 (Kee 2009; Yang et al. 2014). Therefore, to determine whether Id 1 might initiate cardiogenic mesoderm formation by inhibiting E proteins (Fig. 4A), we tested whether siRNAs directed against the three E proteins either alone or in combination (seven combinations) would promote $K d r$-eGFP fluorescence at day 6 of differentiation as above. All combinations of siRNAs that contained siTcf3 promoted cardiogenic mesoderm differentiation (approximately fourfold over siControl) (Fig. 4B-D). Although these studies implicate Tcf 3 as a relevant target of Id1, siTcf 3 was significantly less potent than either Id1 overexpression or siAcvr1b transfection, suggesting that additional targets are involved in inducing Kdr-eGFP $P^{+}$cells. Therefore, we screened all 104 members of the class II family of bHLH transcription factors (e.g., MyoD, NeuroD, myogenin, etc.) by an analogous approach, but none had any effect on cardiogenic mesoderm formation (data not shown).

Next, we tested whether Id1 might mediate part of its procardiogenic mesoderm activity by down-regulating antagonists of cardiogenic mesoderm formation. Such genes should be among those down-regulated in response to the procardiogenic mesoderm actions of siAcvrlb at day 4 of
A
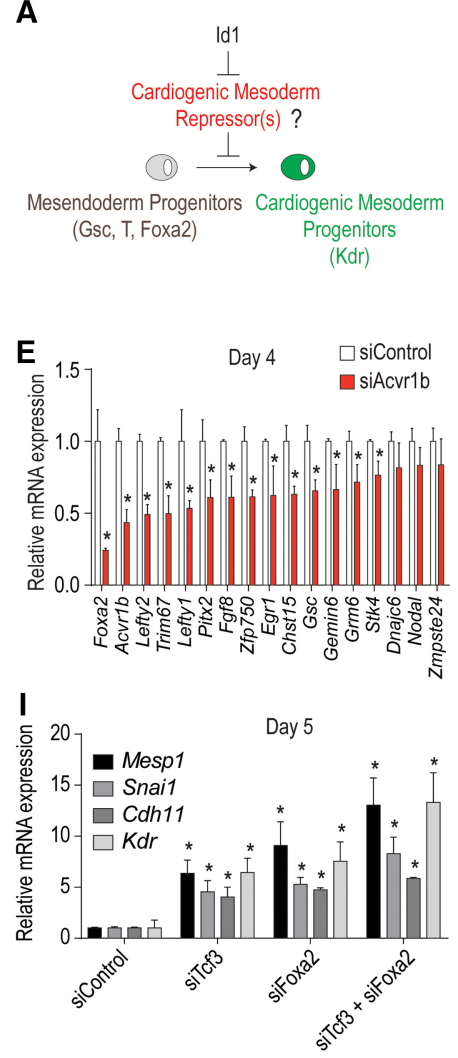
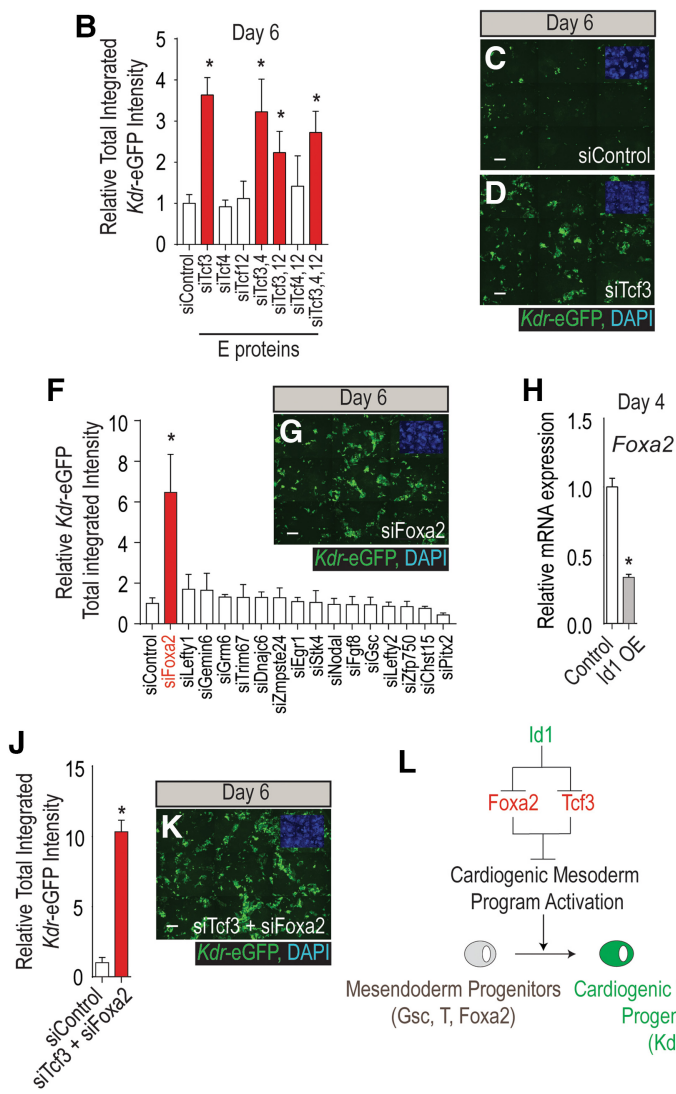

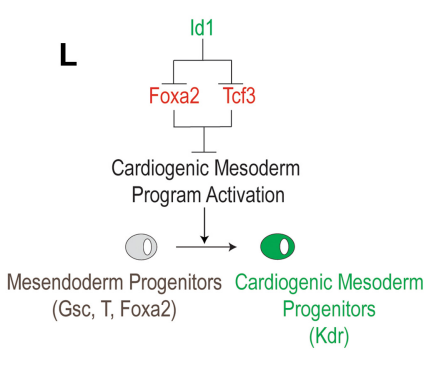

Figure 4. Id1 promotes cardiogenic mesoderm differentiation by inhibiting Tcf3 and Foxa2. (A) Schematic predicting that Id1 mediates its procardiogenic effect by targeting and inhibiting repressors of cardiogenic mesoderm differentiation. (B) siRNA-mediated functional screen evaluating the role of $E$ proteins $(T c f 3, T c f 4$, and $T c f 12)$ in repressing cardiogenic mesoderm differentiation. The diagram shows the fluorescence quantification of $K d r$-eGFP in response to all seven possible siRNA combinations and siControl. $(C, D)$ Representative immunofluorescence images of $K d r$-eGFP at day 6 of differentiation from mESCs transfected at day 3 with siControl $(C)$ and siTcf3 (D). Bar, $50 \mu \mathrm{m}$. (E) qRT-PCR validation showing that 17 genes are down-regulated at day 4 in response to siAcvr1b as compared with siControl $24 \mathrm{~h}$ after transfection. $(F, G)$ siRNA-mediated functional screen evaluating whether downstream targets of Acvr1b signaling are involved in the repression of cardiogenic mesoderm differentiation. $(F)$ The diagram shows the fluorescence quantification of $K d r$ eGFP, where only a siRNA directed against siFoxa2 is able to promote cardiogenic mesoderm differentiation. $(G)$ Representative $K d r$ eGFP immunofluorescence images of siFoxa2. Bar, $50 \mu \mathrm{m}$. $(H)$ qRT-PCR shows that Foxa2 expression is down-regulated in $I d 1$-overexpressing mESCs as compared with control. (I-K) qRT-PCR for cardiogenic mesoderm markers (Mesp1, Snai1, Cdh11, and Kdr) showing that the cotransfection of siFoxa2 and siTcf3 further enhances cardiogenic mesoderm differentiation as compared with siTcf3 or siFoxa2 alone (shown in $I$ ). The diagram shows the fluorescence quantification of $K d r$-eGFP $(J)$ and a representative image $(K)$ of the siTcf3 + siFoxa2 condition. Bar, $50 \mu \mathrm{m}$. $(L)$ Model showing Id1's repressive role on Tcf3 and Foxa2 activity to promote cardiogenic mesoderm differentiation. 
differentiation. Of the 53 genes identified in the microarray (Supplemental Table S1), 17 were confirmed by RT-qPCR to be robustly down-regulated by siAcvrlb (Fig. 4E). Next, we tested whether siRNA-mediated knockdown of any of these 17 genes would be sufficient to promote $K d r$-eGFP ${ }^{+}$cardiogenic mesoderm formation. Strikingly, siRNA to only one gene, encoding the forkhead transcription factor Foxa2, was sufficient to induce $K d r-\mathrm{eGFP}^{+}$mesoderm (Fig. 4F,G). Although Id1 is not known to physically interact with forkhead transcription factors, overexpression of Id 1 strongly decreased the abundance of Foxa2 transcripts in the cells relative to controls (Fig. 4H), suggesting that Id1 indirectly inhibits Foxa2 gene expression.

Consistent with our hypothesis, Tcf3 and Foxa2 knockdowns (Supplemental Fig. S3A,B) derepressed cardiogenic mesoderm gene expression (Mesp1, Snai1, Cdh11, and $K d r)$ (Fig. 4I), and the combined knockdown of Tcf3 and Foxa2 further enhanced cardiogenic mesoderm differentiation efficiency, suggesting that both genes might act in a nonredundant manner during this process (Fig. 4I-K). Finally and in accordance with our molecular model, Tcf3 transcripts are ubiquitously expressed and overlap with Id1 in the most proximal region of the embryo at E6.5, while, in contrast, Foxa2 transcripts are localized in the anterior primitive streak in a domain that is more distal and nonoverlapping with Id1 (Supplemental Fig. S3C,D). We conclude that Id 1 activates the cardiogenic program by inhibiting Tcf3 protein activity while suppressing Foxa2 transcription (Fig. 4L).

\section{Id proteins promote cardiogenic mesoderm formation in vivo}

Next, we used Xenopus embryos to test whether Id genes can promote cardiogenic mesoderm formation in vivo. Equatorial and hemilateral injection of Xid2 mRNA (Fig.
5A), which is the closest ortholog to mouse Id1 (Fig. 5B), caused a dramatic expansion of Xbra $(74 \%, n=105)$ (Fig. 5C,E) and Xmespb (78\%, $n=132)$ (Fig. 5D,F) expression domains (marking mesoderm) in gastrula stage embryos (Nieuwkoop and Faber stage 10.5). To determine whether an expanded $\mathrm{Xbra}^{+}, \mathrm{Xmespb}^{+}$, domain marks an increase in cardiogenic mesoderm formation, $X n k \times 2.5$ expression was examined at tail bud stage (stage 25). Strikingly, Xid2 overexpression caused an expansion of Xnkx2.5 expression $(68 \%, n=88)$ (Fig. 5G-I), while, in contrast, noncardiac mesoderm such as skeletal muscle $(X \mathrm{mlc})$ was not expanded (66\%, $n=30$ ) (Supplemental Fig. S4A-C). Taken together, these data indicate that Xid2, like mammalian Id1 in ESCs, specifically promotes the formation of mesoderm progenitors that are primed to differentiate toward cardiac lineages rather than other mesoderm lineages.

\section{Id genes are essential for early mammalian heart formation in vivo}

Our gain-of-function experiments show that Id proteins are sufficient to direct the formation of cardiac mesoderm both in vitro and in vivo, so we next asked whether Id proteins are normally required for this process. A previous study (Fraidenraich et al. 2004) found that deleting three out of the four Id genes (Id1,2,3 triple gene knockout) caused complex cardiac defects but did not ablate the heart in these embryos, thereby indicating that earlier cardiac specification could still occur. Given the functional similarity of Id family members (Lyden et al. 1999; Fraidenraich et al. 2004; Kee and Bronner-Fraser 2005; Niola et al. 2012, 2013), we reasoned that either redundant or compensatory activity of Id 4 might allow heart formation to occur in triple-knockout embryos. To test this hypothesis, we genetically ablated all four Id gene members using a CRISPR/Cas9 genome-editing strategy in mouse embryos. To increase the probability of null allele
A
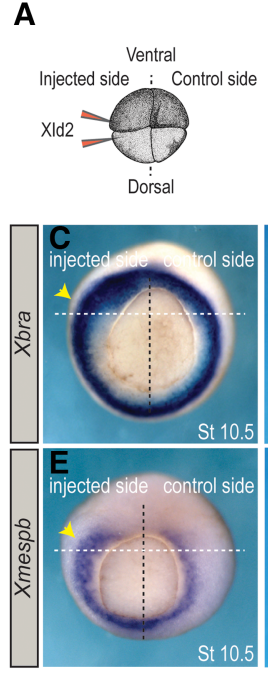

B

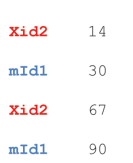

mId1

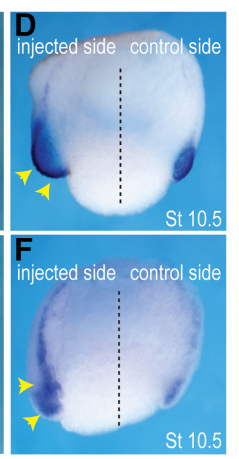

HLH domain (79\% identical, 93\% positives)

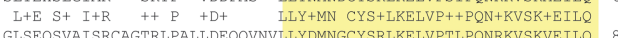
GLSEQSVAISRCAGTRLPALLDEQQVNVLLYDMNGCYSRLKELVPTLPQNRKVSKVEILQ

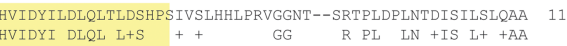
$\begin{array}{lllll}\text { HVIDYI DLQI L+S }++ & \text { GG } & \text { R PL } & \text { LN +IS L+ +AA } & \\ \text { HVIDYIRDLQLELNSESEVGT---_--TGGRGLPVRAPLSTLNGEISALAAEAA } & 137\end{array}$

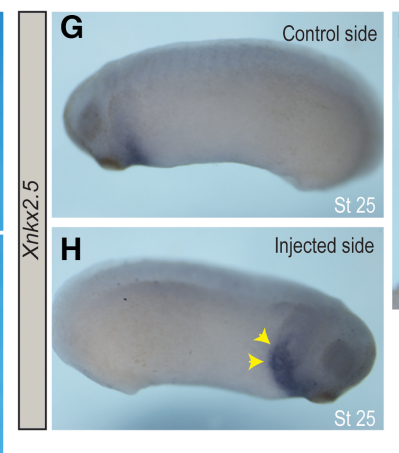
SLTEHSLGIAR---SKTP--VDDPMS--LLYNMNDCYSKLKELVPSIPQNKKVSKMEILQ 66

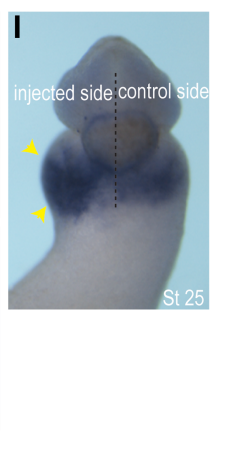

Figure 5. Id proteins promote cardiogenic mesoderm formation in vivo. (A) Xid2 mRNA was injected equatorially into two blastomeres on one side of four-cell stage embryos. (B) The mouse HLH domain of Id1 (NP_034625.1) was aligned and compared with all Xenopus laevis HLH (yellow) domains of id proteins using the Protein Blast tool (https://blast.ncbi.nlm.nih.gov). With $79 \%$ of identical amino acids, Xid2 (NP_001081902.1) (A) is the closest ortholog to Id1. $(C-F)$ Unilaterally injected embryos (as in A) cultured to gastrula stage (stage $10.5)$ in whole mount $(C, E)$ or transversely bisected $(D, F)$ and probed for mesoderm marker Xbra $\quad(C, D)$ and cardiogenic mesoderm Xmespb $(E, F)$ expression. Yellow arrowheads indicate expansions of both the Xbra and $X$ mespb domains in the Xid1-injected side. $(G-I)$ Unilaterally injected embryos cultured to early tail bud stage (stage 25) in whole mount and probed for Xnkx2.5 expression. Yellow arrowheads indicate an expansion of the Xnkx2.5 domain in the Xid2-injected side of the embryo. 
generation, each Id gene was targeted by two single-guide RNAs (sgRNAs) directed against the ATG and the beginning of the HLH domain, respectively. Twenty-four embryos (ranging from E7.75 to E8.75) collected from three independent zygote injection sessions were subjected to genotyping by DNA sequencing and cardiac phenotype assessment by in situ hybridization (Fig. 6A). DNA sequencing results show that despite widespread mosaicism, 320 $(90.7 \%)$ of the 353 alleles detected were null (elimination of the HLH domain reading frame), 24 (6.8\%) were inframe mutations, and only nine $(2.5 \%)$ were wild type. Only seven out of 24 embryos harbored one or more wild-type alleles, while 17 out of 24 embryos harbored no wild-type alleles (Supplemental Fig. S5). Importantly, no off-target mutagenesis was detected in the top eight predicted off-target sites (see the Supplemental Material; http://crispr.mit.edu).

The phenotypic assessment at E7.75 showed that two markers of early cardiac precursors, Smarcd3 and Tbx5, were absent from the most anterior and medial region of the cardiac crescent that gives rise to the heart tube $\mid n=$ 9/11; embryos \#21, \#23, and \#24 for Smarcd3 and \#1-\#4,
\#7, \#8, \#10, and \#13 for Tbx5) (Fig. 6B-I; Supplemental Fig. S6A-D; see Supplemental Fig. S5 for genotype information). In contrast, expression of these markers was maintained in two lateral domains of the mesoderm posterior to the heart tube-forming region, suggesting that these posterior cardiac progenitors could differentiate and migrate appropriately. At E8.25, when the heart tube has normally formed, in situ hybridization for the cardiac marker $N k \times 2.5$ revealed an absence of heart tube formation in Id1-4 mutants (Fig. 6J-M; Supplemental Fig. S6E,F). Histological sectioning confirmed the absence of anatomical heart tube formation (Fig. $6 \mathrm{~K}^{\prime}, \mathrm{M}^{\prime}$ ). At E8.75, when the heart begins to loop, analysis of Nkx2.5 (Fig. 6O$\mathrm{Q})$ and the first heart field marker Tbx5 (Fig. 6R-U) confirmed the absence of hearts in quadruple-knockout embryos ( $n=10 / 13$; embryos \#14, \#15, \#17, and \#22 for Nkx2.5 and \#5, \#6, \#9, \#11, \#12, and \#13 for Tbx5) (Fig. $6 \mathrm{~L}, \mathrm{M}, \mathrm{P}, \mathrm{Q}, \mathrm{T}, \mathrm{U})$. Finally and consistent with our initial hypothesis of functional redundancy between Id family members, embryos harboring at least one Id4 wild-type allele can still form a heart tube that loops, albeit abnormally, as compared with controls ( $n=3 / 13$; embryos \#18-\#20)

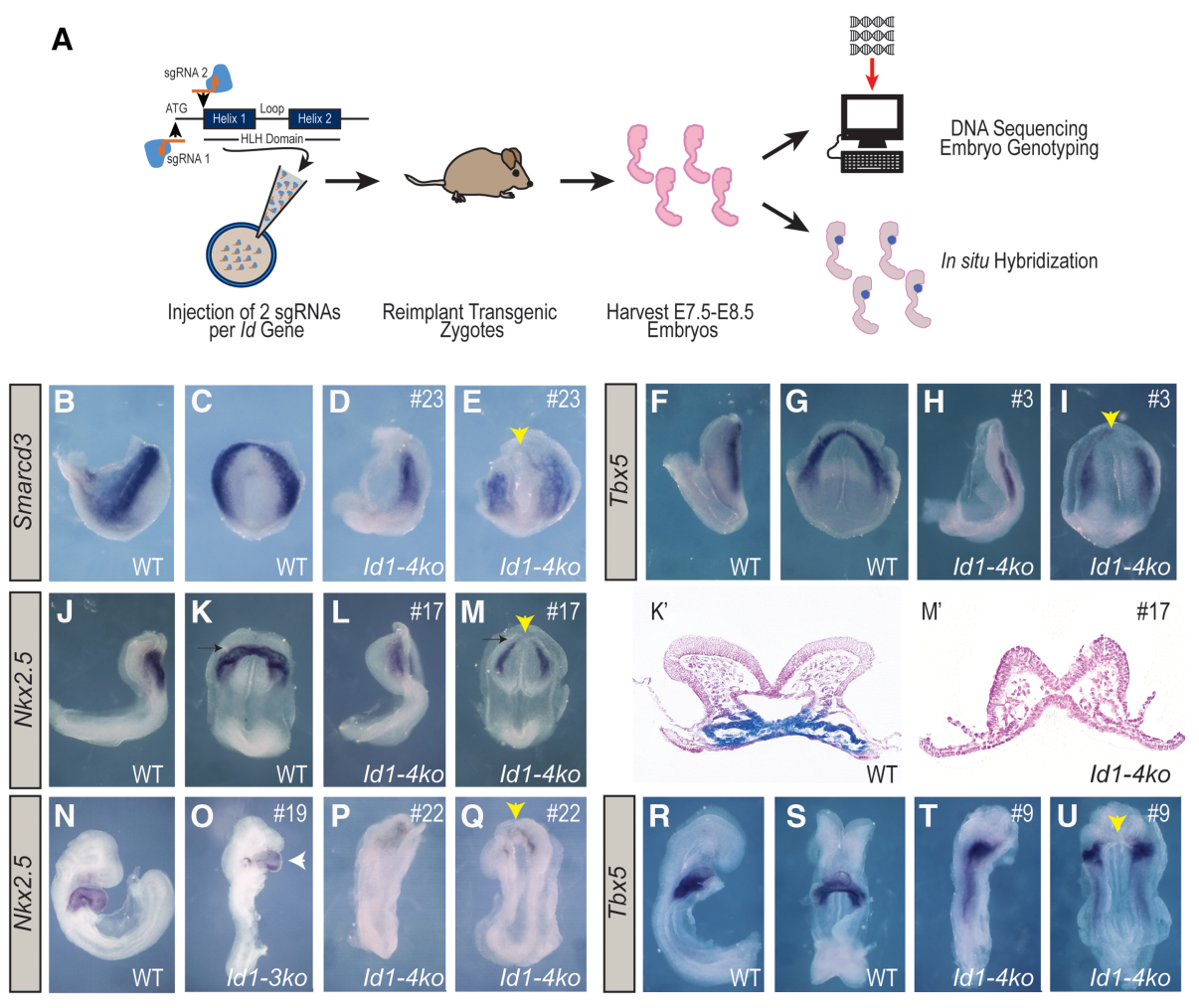

Figure 6. Id genes are essential for early heart formation. (A) Schematic illustrating the generation and analysis of Id1-4 mutant embryos using CRISPR/Cas9 technology. Two sgRNAs per gene (targeting the translational start site and the HLH domain) were injected into single-cell mouse zygotes alongside Cas9 mRNA. Zygotes were reimplanted and harvested at stages E7.5-E8.5. Resulting embryos were genotyped by DNA deep sequencing, and cardiac gene expression was assessed via whole-mount in situ hybridization. (B-U) In situ hybridization results from the most severe Id1-4 mutants—compared with wild type (individual mutants are marked by \#)—plus one less-affected mutant $(O)$; analysis of Smarcd3 at E7.75 (B-E), Tbx5 at E8.0 (F-I), Nkx2.5 at E8.25 (J-M; plus transverse sections through the heart tube-forming region $\left.\left[K^{\prime}, M^{\prime}\right]\right), N k x 2.5$ at E8.5 (N-Q), and Tbx5 at E8.5 (R-U). (Yellow arrowheads) Missing heart tube (or missing heart tube-forming region at cardiac crescent stages) in Id1-4 mutants; (white arrowhead) malformed heart tube; (black arrows) the plane of transverse sectioning through the heart tube-forming region; (black dashed arrows) posterior-lateral cardiac regions. See the Supplemental Material for detailed sequencing results of mutant embryos. 
(Fig. 6N,O). Collectively, these results demonstrate that the Id family of genes is required for the specification of heart tube-forming CMPs and their subsequent assembly.

\section{Discussion}

\section{Molecular control of cardiogenic mesoderm specification}

Unraveling the molecular mechanisms controlling cardiogenic mesoderm specification is crucial for our understanding of how heart formation is normally initiated during embryonic development and for our ability to generate developmentally relevant cardiac progenitors for therapeutic and cardiac disease modeling purposes. This study reveals that cardiogenic mesoderm specification is tightly regulated in bipotent mesendoderm progenitors by an antagonistic interplay between Id proteins and the Acvr1b signaling pathway. High Acvr1b signaling represses the expression of $I d$ genes and biases mesendoderm progenitors to differentiate toward endoderm. Conversely, attenuation of Acvr1b signaling in these cells derepresses $I d$ gene transcription that in turn promotes cardiogenic mesoderm specification (Fig. 7).

A central finding of this study is the ability of Id proteins to override proendoderm cues (induced by high Acvr1b signaling) to promote cardiogenic mesoderm differentiation instead. The functional dominance of Id proteins over Acvr1b signaling also implies that molecules controlling the spatial and quantitative distribution of Id proteins are likely to be crucial regulators of cardiogenic mesoderm formation. Consistent with our model, BMP signaling directly activates Id1 transcription (Hollnagel et al. 1999; Katagiri et al. 2002; Korchynskyi and ten Dijke 2002; Lopez-Rovira et al. 2002) and is both necessary and sufficient to induce cardiogenic mesoderm formation (Beppu et al. 2000; Yang et al. 2008; Paige et al. 2012). Conversely, the finding that Acvr1b signaling represses $I d 1 / 3$ gene expression is consistent with the ability of a small molecule inhibitor of the Nodal receptor (SB431542) to up-regulate $I d 1$ transcripts in mESCs (Galvin et al. 2010) and reinforces the role of Acvr1b signaling in opposing cardiac cell fate acquisition during gastrulation. In summary, high Id protein levels in mesendoderm progenitors constitute a dominant molecular cue that is sufficient to trigger and orchestrate cardiogenic mesoderm specification in vertebrates.

\section{Positioning Id genes in the context of Mesp1 procardiogenic activity}

Several transcription factors have been shown to be essential for cardiac development (Olson 2006; Bruneau 2013). Among them, Mesp1 is the earliest expressed and is sufficient to directly promote cardiac specification in mesoderm progenitors (Saga et al. 1996; Bondue et al. 2008; David et al. 2008; Chan et al. 2013). Importantly, our gain-of-function experiments show that Id1/Xid2 is sufficient to direct Mesp1/Xmespb expression in both mESCs and hESCs as well as in Xenopus embryos and subsequently promote cardiogenic mesoderm differentiation. These observations suggest that Id proteins exert at least part of their procardiogenic effect by up-regulating Mesp

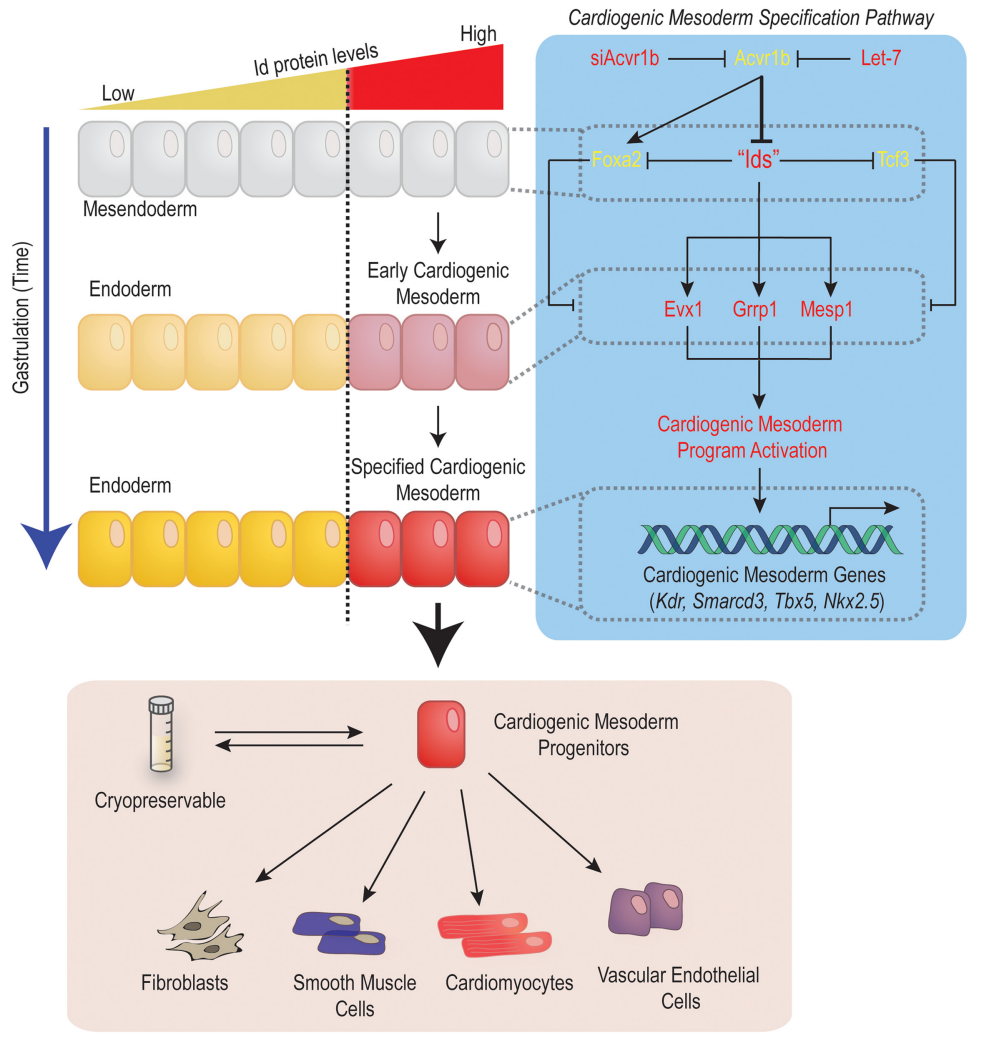

Figure 7. Id genes orchestrate cardiogenic mesoderm differentiation in vertebrates. Id genes control the activation of the cardiogenic mesoderm differentiation program in mesendoderm progenitors by inhibiting the activity of repressors (Tcf3 and Foxa2) while promoting the expression of activators of cardiogenic mesoderm differentiation (Evx1, Grrp1, and Mesp1). The Id-controlled network induces cardiogenic mesoderm (Mesp1 and $K d r$ ) differentiation from pluripotent cells. Id1-induced CMPs generated from pluripotent stem cells are cryopreservable and spontaneously form contracting cardiomyocytes $(\sim 70 \%)$ as well as vascular endothelial cells, smooth muscle, and fibroblasts. 
genes. Since Id proteins do not directly bind DNA (Benezra et al. 1990; Roschger and Cabrele 2017), we propose that the up-regulation of Mesp genes is likely to involve the inhibition of proteins that repress Mesp transcription. Consistent with our hypothesis, siRNA-mediated knockdown of two transcription factors, Tcf3 and Foxa2, is sufficient to up-regulate Mesp 1 expression and promote $\mathrm{Kdr}^{+}$ mesoderm formation. Mechanistically, Tcf3 is a wellcharacterized target of Id proteins (for review, see Yang et al. 2014) and is also known to interact with the Smad/ Foxh1 complex to regulate Smad2/3-dependent transcription in mesendoderm progenitors (Yoon et al. 2011; Wills and Baker 2015). In turn, the potent endoderm determinant Foxa2 (Stainier 2002; Viotti et al. 2014), is expressed in mesendoderm progenitors in a Smad2-dependent manner in mouse embryos (Vincent et al. 2003), suggesting that Tcf3 might cooperate with $\operatorname{Smad} 2$ to regulate Foxa2. Considering these functions, we suggest that the Id-driven blockade of Tcf3 and Foxa2 function drives two concomitant processes in mesendoderm progenitors: (1) the prevention of Acvr1b/Smad2-driven endoderm differentiation and (2) the activation of cardiac mesoderm specification via Mesp gene up-regulation.

\section{Id genes are essential for the specification of heart tube-forming progenitors}

Heart formation in mammals involves a complex sequence of cell fate decisions and morphogenetic events that interdependently contribute to generate a four-chambered heart. Although we cannot completely rule out the possible contribution of endoderm morphogenetic defects to the absence of heart tube formation in Id1-4 mutant embryos, several lines of evidence support that these defects directly result from impaired cardiac mesoderm specification due to the loss of Id protein activity in mesendoderm progenitors: (1) A closed foregut can be observed in the most anterior part of the embryo, where the heart tube normally forms (Supplemental Fig. S7D), suggesting that the endoderm could form and undergo morphogenesis. (2) Overall staining of cardiac mesoderm markers (Smarcd3, Tbx5, and Nkx2.5) is diminished in Id1-4 mutant embryos as compared with controls (Fig. $6 \mathrm{~B}-\mathrm{N}$ ), which suggests that fewer cardiac progenitors have formed. (3) siRNA-mediated loss of Id1 function impairs cardiac mesoderm formation in mESCs (Fig. 1J,K), a process that is de facto independent of endoderm morphogenesis in vitro. (4) Gain-of-function experiments show that Id proteins are sufficient to direct cardiac mesoderm specification in ESCs and Xenopus and expand the cardiac primordium in Xenopus embryos (Figs. 2, 3). Collectively, these observations suggest that the Id protein's requirement for anterior cardiac mesoderm specification is independent of endoderm differentiation and morphogenesis during early heart formation.

It is well described that most of the heart myocardium in mammals derives from two distinct populations of cardiac progenitors, referred to as heart fields (Kelly et al. 2001; Cai et al. 2003; Meilhac et al. 2004, 2015). However, it is not known whether similar or distinct molecular mechanisms regulate cardiac specification in these two cell populations. Our loss-of-function results showed that embryos lacking functional Id1-4 genes fail to express cardiogenic mesoderm markers (Smarcd3, Tbx5, and Nkx2.5) in the most anterior region of the cardiac crescent at E7.75 and subsequently develop without forming a heart tube. In contrast, expression of these genes is maintained in the posterior region of the cardiac crescent within the splanchnic cardiac mesoderm (Supplemental Fig. S7A-E). Collectively, these observations suggest that only the most anterior subset of cardiac progenitors require Id1-4 activity for their specification. Consistent with our findings, Lescroart et al. (2014) have shown recently that early Mesp1expressing mesoderm progenitors (around E6.5), which contribute to first heart field derivatives, express high levels of Id1. In contrast, late Mesp1-expressing cells (around E7.5), which contribute to second heart field derivatives, express low levels of Id1 (see the Supplemental Material of Lescroart et al. 2014). Moreover, we show that human iMPs upregulate first heart field markers (HCN4 and $T B X 5)$ during cardiac differentiation, while second heart field markers (ISL1 and SIX2) are down-regulated (Supplemental Fig. S8A-F). Thus, we propose that Id genes normally specify first heart field progenitors that subsequently form the early heart tube. These findings also imply that cardiogenic mesoderm specification is not a singular process and can be initiated in an Id-dependent (first heart field progenitors) or Id-independent (posterior cardiac progenitors) manner during embryonic development.

\section{Id1-induced CMPs: a promising new technology for cardiac regenerative medicine and disease modeling}

Recent studies have validated the concept of using ESCderived cardiac progenitor transplantation to improve heart function post-injury in rodents, sheep, and nonhuman primate models (Menasche et al. 2015). Although promising, the use of these cells for therapeutic regeneration has not advanced substantially, largely because of the challenge of generating large numbers of defined cardiac progenitors from stem cells. This study demonstrates that simple overexpression of Id1 in hESCs (or human induced pluripotent stem cells [hiPSCs]) (data not shown) is sufficient to generate large amounts $\left(>10^{8}\right.$ cells per batch) of cryopreservable and bona fide CMPs with remarkable abilities to spontaneously differentiate into beating cardiomyocytes ( $70 \%$ efficiency). These combined properties enable two major applications for Id1-programmed progenitors: (1) as a promising transplantable cell population to test for cardiac regenerative purposes after myocardial injury and (2) as a novel source of cells enabling large-scale production of hESC- or hiPSC-derived cardiomyocytes suitable for in vitro studies of cardiomyocyte physiology.

\section{Materials and methods}

All animal handling and care followed the National Institutes of Health Guide for Care and Use of Laboratory Animals. The experimental protocols were approved by the Institutional Animal Care and Use Committees of the Sanford Burnham Prebys 
Medical Discovery Institute (SBP) (Xenopus) or the University of California at San Diego (mice).

mESC culture

Kdr-eGFP mESCs (Ema et al. 2006) were maintained in DMEM high-glucose (HyClone) medium supplemented with $10 \%$ fetal bovine serum (FBS), $1 \mathrm{mM}$ sodium pyruvate (Sigma), 1× MEM nNEAA (Gibco), 2 mM L-glutamine (Gibco), $100 \mathrm{U} / \mathrm{mL}$ penicillin-100 $\mu \mathrm{g} / \mathrm{mL}$ streptomycin, (HyClone), $50 \mu \mathrm{M} \beta$-mercaptoethanol (Sigma), and $1000 \mathrm{U} / \mathrm{mL}$ LIF (Millipore). For differentiation, mESCs were seeded in 10-cm low-attachment tissue culture dishes at a density of $10^{6}$ cells per dish in a chemically defined medium (CDM) (Gadue et al. 2006), where they formed embryoid bodies (EBs) over a period of $2 \mathrm{~d}$. At day 2, EBs were then dissociated using $0.25 \%$ Trypsin EDTA (GIBCO), washed in PBS, and replated in CDM supplemented with $50 \mathrm{ng} / \mathrm{mL}$ recombinant human Activin A (R\&D Systems, 338-AC-050) in 10-cm low-attachment tissue culture dishes.

\section{Kdr-eGFP assay}

On day 3 of differentiation, EBs were collected and dissociated using $0.25 \%$ Trypsin EDTA; $10^{4}$ cells per well were plated in $100 \mu \mathrm{L}$ of CDM containing $50 \mathrm{ng} / \mathrm{mL}$ recombinant human Activin A in gelatin-coated 384-well optical tissue culture plates (Greiner Bio-One) and prespotted with $25 \mathrm{nM}$ siRNAs in $0.2 \mu \mathrm{L}$ of Lipofectamine RNAiMax $+14.8 \mu \mathrm{L}$ of Opti-Mem I (Gibco). Fixation was performed at day 6 using $4 \%$ paraformaldehyde. Next, wells were imaged using an HT microscope (ImageXPress, Molecular Devices), and fluorescence was quantified using a custom method developed in MetaXpress analysis software (Molecular Devices) to determine the integrated pixel intensity of $K d r$-eGFP.

\section{hESC culture}

The H9 hESC line (WA09) was supplied by WiCell Research Institute. $\mathrm{H} 9$ cells were routinely maintained in mTeSR 1 medium (Stem Cell Technologies, 05850) on growth factor-reduced Matrigel at $9 \mu \mathrm{g} / \mathrm{cm}^{2}$ and passaged every $4 \mathrm{~d}$ using ReLeSR (Stem Cell Technologies, 05872). hESCs were cultured for at least five passages before beginning differentiation. Cells were maintained with $2.5 \mathrm{~mL}$ of medium per $9.6 \mathrm{~cm}^{2}$ of surface area or equivalent. All pluripotent cultures were negative for mycoplasma contamination as routinely tested using a MycoAlert kit (Lonza).

\section{Lentivirus preparation}

Large-scale lentivirus production was performed by the Viral Vector Core Facility at the SBP. Briefly, three plasmids, including lentivector, pCMVDR8.74, and pMD2.G, were cotransfected into HEK293T cells at a ratio of 3:2:1. UltraCulture serum-free medium (Lonza) supplemented with $1 \mathrm{mM}$ L-glutamine (Life Technologies) was used to refeed transfected cells, and the supernatant was collected every $24 \mathrm{~h}$ from day 2 to day 4 after transfection. All viral supernatant was pooled and filtrated through $0.22-\mu \mathrm{m}$ pores followed by concentration and purification using $20 \%$ sucrose gradient ultracentrifugation at $21,000 \mathrm{rpm}$ for $2 \mathrm{~h}$. The pellet containing concentrated viral particles was resuspended in PBS, aliquoted, and kept at $-80^{\circ} \mathrm{C}$ for long-term storage.

Generation of transgenic cell lines (mESCs and hESCs)

We applied the following modifications to $\mathrm{pCDH}-\mathrm{CMV}$ vector (System Biosciences, CD511B-1): The CMV promoter driving the expression of the MCS was replaced by the Ef1a promoter to ensure robust expression in ESC stages, and the Ef1a-CopGFP cassette was replaced by a $p g k$-puro cassette to enrich for infected clones.mESCs with $K d r$-eGFP (Ema et al. 2006) were infected with all possible combinations of high-titer lentiviruses (modified pCDH-CMV) (see above) overexpressing Id1, Evx1, or Grrp1 and subsequently grown under continuous $2 \mu \mathrm{g} / \mathrm{mL}$ puromycin selection (Acros, 227420100).

Similarly, H9 hESCs were infected with Id1-overexpressing lentivirus and selected with $6 \mu \mathrm{g} / \mathrm{mL}$ puromycin.

\section{Generation of Id1-induced CMPS}

Mouse Id1-induced CMPs Id1-overexpressing mESCs were grown and differentiated as wild-type mESCs in the presence of $2 \mu \mathrm{g} /$ $\mathrm{mL}$ puromycin. At day 3, cells were collected and dissociated with $0.25 \%$ Trypsin EDTA (Gibco), Trypsin was inactivated with $10 \%$ FBS-containing medium, and cells were washed in PBS and resuspended in CDM supplemented with $300 \mathrm{ng} / \mathrm{mL}$ recombinant human Activin A $+2 \mu \mathrm{g} / \mathrm{mL}$ puromycin. Cells $\left(10^{7}\right)$ were replated onto a $15-\mathrm{cm}$ gelatin-coated tissue culture dish into $30 \mathrm{~mL}$ of $\mathrm{CDM}+300 \mathrm{ng} / \mathrm{mL}$ recombinant human Activin A $+2 \mu \mathrm{g} / \mathrm{mL}$ puromycin and cultured for $3 \mathrm{~d}$. At day 6, cells were collected and frozen in freezing medium (10\% DMSO, $20 \%$ FBS, $70 \%$ DMEM high glucose [Hyclone]) at a density of $3 \times 10^{6}$ to $5 \times 10^{6}$ cells per vial and stored in liquid nitrogen.

Human Id1-induced CMPs hESCs were dissociated using $0.5 \mathrm{mM}$ EDTA (Life Technologies) in PBS without $\mathrm{CaCl}_{2}$ or $\mathrm{MgCl}_{2}$ (Corning, 21-040-CV) for $7 \mathrm{~min}$ at room temperature. Cells were plated at $3 \times 10^{5}$ cells per well of a 12 -well plate in mTeSR 1 medium (Stem Cell Technologies) supplemented with $2 \mu \mathrm{M}$ thiazovivin (Selleck Chemicals) for the first $24 \mathrm{~h}$ after passage. Cells were fed daily for 3-5 d until they reached $\geq 90 \%$ confluence, at which time they were washed with PBS, and the medium was changed to basal differentiation medium (BDM) consisting of RPMI 1640 medium (Life Technologies, 11875-093) and B27 supplement minus insulin (Life Technologies, A1895601). For the first 24-h differentiation period, the BDM was supplemented with $300 \mathrm{ng} /$ $\mathrm{mL}$ recombinant human Activin A and $2 \mu \mathrm{g} / \mathrm{mL}$ puromycin (Acros, 227420100). After $24 \mathrm{~h}$, this medium was replaced with basic BDM supplemented with $6 \mu \mathrm{g} / \mathrm{mL}$ puromycin. BDM +6 $\mu \mathrm{g} / \mathrm{mL}$ puromycin was replaced every $48 \mathrm{~h}$. At day 5 , cells were collected and frozen as described above.

\section{Differentiation of cryopreserved Id1-induced CMPS}

To resume differentiation, CMPs (mouse or human) were thawed for $3 \mathrm{~min}$ in a $37^{\circ} \mathrm{C}$ water bath, washed, resuspended in $2 \mu \mathrm{M}$ $\mathrm{BDM}^{+} \mathrm{hESC}$ recovery supplement (Stemgent) for human Idl-induced CMPs or $2 \mu \mathrm{M} \mathrm{CDM}^{+}$hESC recovery supplement for mouse Id1-induced MMPs, and plated onto Matrigel-coated 384-well culture plates (Greiner Bio-One) at a cell density of 25,000 cells per well. Medium (BDM or CDM) was replaced every other day until day 15 of differentiation.

SiRNAs

siRNAs from Figures $1 \mathrm{~F}$ and $3 \mathrm{~F}$ were cherry-picked at the International Centre for Genetic Engineering and Biotechnology (Trieste, Italy) from the mouse genome-wide siGENOME SMARTpool library from Dharmacon and transfected at a final concentration of $12.5 \mathrm{nM}$. All remaining siRNAs were purchased from Life Technologies (silencer select siRNAs) and transfected at a final concentration of $25 \mathrm{nM}$ : siControl (AM4611), siEvx1 (s65742), siFoxa2 (s67627), siGrrp1 (s91214), siId1 (s68006), siTcf3 (s74856), siTcf4 (s74829), and siTcf12 (s74811). 
Immunostaining for cell culture and cardiovascular lineage quantification

Cells grown on gelatin-coated 384-well plates (Greiner Bio-One) were fixed using $4 \%$ paraformaldehyde and immunostained by incubating in block solution $(10 \%$ horse serum, $0.5 \%$ Triton X-100, $0.01 \%$ gelatin in phosphate-buffered saline [PBS] [Cellgro]) for 30 min at room temperature followed by incubation with antibodies directed against Foxa2 (Santa Cruz Biotechnology, sc-6554), Pecam1 (Santa Cruz Biotechnology, sc-1506), Actc1 (Sigma, A7811), and CDH5 (R\&D Systems, AF938) for $1 \mathrm{~h}$ at room temperature in the block solution. The cells were then washed three times with PBS and incubated with Alexa-conjugated secondary antibodies(Life Technologies) in the block solution for $1 \mathrm{~h}$ at room temperature. The cells were then washed three times with PBS and stored in $50 \%(\mathrm{v} / \mathrm{v})$ glycerol in PBS. Next, wells were imaged using a HT microscope (ImageXPress, Molecular Devices), and fluorescence was quantified using a custom method developed in MetaXpress Analysis software (Molecular Devices) to determine the percentages of ACTC1-, TAGLN-, and CDH5-positive cells.

\section{$R T-q P C R$}

Total RNA was extracted with a miRVana isolation kit (Ambion, AM1540) and reverse-transcribed to cDNA with a QuantiTect reverse transcription kit (Qiagen) according to the manufacturer's instructions. cDNA samples synthesized from $1 \mu \mathrm{g}$ of total RNA were subjected to RT-qPCR with the $7900 \mathrm{HT}$ Fast realtime PCR system (Applied Biosystems) using the iTaq SYBR Green Supermix with ROX (Bio-Rad). Primer sequences are listed in Supplemental Table S2. The data were analyzed with the $\Delta \Delta \mathrm{Ct}$ method, applying $\beta$-Actin as a normalization control.

\section{Microarray experiment and analysis}

siControl or siAcvrlb was transfected at day 3 in differentiating mESCs. Total RNA (500 ng) was collected at day 4 and hybridized on MouseRef-8 v2.0 Expression BeadChip (25,600 transcripts; Illumina). BeadChips were subsequently washed and developed with Fluorolink streptavidin-Cy3 (GE Healthcare). BeadChips were scanned with an Illumina BeadArray Reader, and hybridization efficiency was monitored using BeadStudio software (Illumina) to measure internal controls built into the Illumina system. Linear models were fitted for each gene using the Bioconductor limma package in R. Moderated $t$ statistics and fold change and the associated $P$-values were calculated for each gene. To account for testing thousands of genes, we reported false discovery rate (FDR)-adjusted values, which were calculated using the Benjamini-Hochberg method.

\section{Flow cytometry}

For live $K d r$-eGFP cells, cells were dissociated using $0.25 \%$ Trypsin EDTA, blocked with 10\% FBS-containing medium, and resuspended in PBS containing 0.5\% FBS (washing buffer) for flow sorting using LSRFortessa or FACSAria flow cytometers (BDBiosciences). For hESCs, day 5 cells were dissociated using 1× TrypLE Express (Gibco), blocked, and washed with PBS containing $0.5 \%$ FBS (washing buffer). Cells were incubated for $20 \mathrm{~min}$ with PE anti-human CD309 (1:100 dilution; BioLegend, 359903) in PBS containing $0.5 \% \mathrm{FBS}$ at $4^{\circ} \mathrm{C}$. Next, cells were washed in washing buffer, fixed for $20 \mathrm{~min}$ in $1 \% \mathrm{PBS}$ :formaldehyde at $4^{\circ} \mathrm{C}$, washed in PBS, and resuspended in washing buffer and processed by flow sorting.

Xenopus laevis embryo culture

Embryos were fertilized in vitro, dejellied in $2 \%$ cysteine- $\mathrm{HCl}$ (pH 7.8), and maintained in 0.1× MMR (Peng 1991). Embryos were staged according to Nieuwkoop and Faber (Nieuwkoop 1967). For gene expression analysis, whole embryos were fixed in MEMFA for in situ hybridization as below.

\section{$m R N A$ injection in $\mathrm{X}$. laevis}

Synthetic capped mRNAs for Xid2 injection were transcribed from pSP64T plasmid using SP6 mMessage kit (Ambion). mRNAs were injected at $125 \mathrm{ng}$ per blastomere in four-cell stage embryos.

In situ hybridization in X. laevis embryos

In situ hybridization for Xbra (Colas et al. 2008) and Xmespb was carried out as described previously (Djiane et al. 2000).

\section{Mouse embryos}

Mouse embryos were dissected into DEPC-treated PBS, fixed overnight in $4 \% \mathrm{PFA}$, and dehydrated into $\mathrm{MeOH}$. In situ hybridization used Id1, Grrp1, Evx1 (cloned into pGEM), and Mesp1 (Saga et al. 1996) probes $\left(60^{\circ} \mathrm{C}\right.$ hybridization) as described in Wilkinson and Nieto (1993). For histology, embryos were embedded in paraffin, $\mathrm{H} \& \mathrm{E}$ stained, and sectioned (5- $\mu \mathrm{m}$ thickness) following standard procedures. Sections were scanned at high magnification (40x) using a Leica Aperio AT2.

\section{CRISPR/Cas9 Id gene editing of mouse embryos}

In order to generate Id1-4 mutant embryos, eight sgRNAs were designed to target sites near the ATG translation initiation site and near the beginning of the HLH domain for each Id gene (see the Supplemental Material) using the tool at http://crispr. mit.edu to ensure maximum specificity. DNA templates for sgRNAs were generated by PCR amplification (Phusion DNA polymerase, New England Biolabs) of ssDNA ultramer oligonucleotides (Integrated DNA Technologies) (see the Supplemental Material); sgRNAs were transcribed from these templates using HiScribe T7 high-yield RNA synthesis kit (New England Biolabs) and purified using Megaclear kit (Life Technologies). For mouse zygote injections, $50 \mathrm{ng} / \mathrm{\mu L}$ Cas 9 mRNA (Life Technologies) and $20 \mathrm{ng} / \mu \mathrm{L}$ each sgRNA were combined in nuclease-free water. Fertilized oocytes were collected from 3- to 4-wk-old superovulated C57Bl6 females (prepared by injecting 5 IU each of pregnant mare serum gonadotropin and human chorionic gonadotropin [Sigma Aldrich]), transferred into M2 medium (Millipore), and injected with the Cas9 mRNA/sgRNA solution into the cytoplasm. Injected embryos were then reimplanted into recipient pseudopregnant ICR female mice. Implanted females were sacrificed 8-9 d after reimplantation; yolk sac DNA was collected for genotyping by PCR (Bioline MyTaq extract kit) (see the Supplemental Material for primer sequences) followed by DNA deep sequencing (Ilumina Nextera kit for library preparation and Illumina HiSeq 1500 for sequencing). Sequences were analyzed, and variant alleles were recorded using Integrative Genomics Viewer (IGV) genome browser (Broad Institute). For off-target analysis, the top eight off-target sites were identified using the tool at http://crispr.mit.edu; these regions were PCR-amplified (see the Supplemental Material for off-target sites and primer sequences) and Sanger-sequenced.

\section{Statistics}

Each experiment represents at least quadruplicate biological replicates per condition. Statistical analysis was performed with unpaired Student's $t$-test $(P<0.05)$. 


\section{Acknowledgments}

We gratefully acknowledge Pedro Aza-Blanc and the Sanford Burnham Prebys Medical Discovery Institute Functional Genomics core (supported by P30 CA030199-30) for screening support. This research was funded by grants from the National Institutes of Health (R01HL113601, R01HL128072, and R01HL130840 to M. Mercola; R44ES023521 to A.C.; P30 AR06130303 to M. Mercola, A.S., and P.L.P.; R01AR056712 and R01AR052779 to P.L.P.; and F31 AR065923-01 to M.T.), the Muscular Dystrophy Association (200845 to A.S.), the Fondation Leducq Transatlantic Alliance (Shapeheart), and the California Institute for Regenerative Medicine (CIRM; RC1-000132 to M. Mercola). S.A. was supported by the CIRM post-doctoral training program (TG2-01162).

\section{References}

Bardot E, Calderon D, Santoriello F, Han S, Cheung K, Jadhav B, Burtscher I, Artap S, Jain R, Epstein J, et al. 2017. Foxa2 identifies a cardiac progenitor population with ventricular differentiation potential. Nat Commun 8: 14428 .

Benezra R, Davis RL, Lockshon D, Turner DL, Weintraub H. 1990. The protein Id: a negative regulator of helix-loop-helix DNA binding proteins. Cell 61: 49-59.

Beppu H, Kawabata M, Hamamoto T, Chytil A, Minowa O, Noda T, Miyazono K. 2000. BMP type II receptor is required for gastrulation and early development of mouse embryos. Dev Biol 221: 249-258.

Birket MJ, Ribeiro MC, Verkerk AO, Ward D, Leitoguinho AR, den Hartogh SC, Orlova VV, Devalla HD, Schwach V, Bellin $\mathrm{M}$, et al. 2015. Expansion and patterning of cardiovascular progenitors derived from human pluripotent stem cells. Nat Biotechnol 33: 970-979.

Bondue A, Lapouge G, Paulissen C, Semeraro C, Iacovino M, Kyba M, Blanpain C. 2008. Mesp1 acts as a master regulator of multipotent cardiovascular progenitor specification. Cell Stem Cell 3: 69-84.

Bruneau BG. 2013. Signaling and transcriptional networks in heart development and regeneration. Cold Spring Harb Perspect Biol 5: a008292.

Buckingham M, Meilhac S, Zaffran S. 2005. Building the mammalian heart from two sources of myocardial cells. Nat Rev Genet 6: 826-835.

Burridge PW, Matsa E, Shukla P, Lin ZC, Churko JM, Ebert AD, Lan F, Diecke S, Huber B, Mordwinkin NM, et al. 2014. Chemically defined generation of human cardiomyocytes. Nat Methods 11: 855-860.

Cai CL, Liang X, Shi Y, Chu PH, Pfaff SL, Chen J, Evans S. 2003. Isl1 identifies a cardiac progenitor population that proliferates prior to differentiation and contributes a majority of cells to the heart. Dev Cell 5: 877-889.

Chan SS, Shi X, Toyama A, Arpke RW, Dandapat A, Iacovino M, Kang J, Le G, Hagen HR, Garry DJ, et al. 2013. Mesp1 patterns mesoderm into cardiac, hematopoietic, or skeletal myogenic progenitors in a context-dependent manner. Cell Stem Cell 12: $587-601$.

Chiapparo G, Lin X, Lescroart F, Chabab S, Paulissen C, Pitisci L, Bondue A, Blanpain C. 2016. Mesp1 controls the speed, polarity, and directionality of cardiovascular progenitor migration. I Cell Biol 213: 463-477.

Colas A, Cartry J, Buisson I, Umbhauer M, Smith JC, Riou JF. 2008. Mix.1/2-dependent control of FGF availability during gastrulation is essential for pronephros development in Xenopus. Dev Biol 320: 351-365.
Colas AR, McKeithan WL, Cunningham TJ, Bushway PJ, Garmire LX, Duester G, Subramaniam S, Mercola M. 2012. Whole-genome microRNA screening identifies let-7 and mir-18 as regulators of germ layer formation during early embryogenesis. Genes Dev 26: 2567-2579.

Collop AH, Broomfield JA, Chandraratna RA, Yong Z, Deimling SJ, Kolker SJ, Weeks DL, Drysdale TA. 2006. Retinoic acid signaling is essential for formation of the heart tube in Xenopus. Dev Biol 291: 96-109.

Costello I, Pimeisl IM, Drager S, Bikoff EK, Robertson EJ, Arnold SJ. 2011. The T-box transcription factor Eomesodermin acts upstream of Mesp1 to specify cardiac mesoderm during mouse gastrulation. Nat Cell Biol 13: 1084-1091.

David R, Brenner C, Stieber J, Schwarz F, Brunner S, Vollmer M, Mentele E, Muller-Hocker J, Kitajima S, Lickert H, et al. 2008. MesP1 drives vertebrate cardiovascular differentiation through Dkk-1-mediated blockade of Wnt-signalling. Nat Cell Biol 10: 338-345.

Devine WP, Wythe JD, George M, Koshiba-Takeuchi K, Bruneau BG. 2014. Early patterning and specification of cardiac progenitors in gastrulating mesoderm. Elife 3: e03848.

Diiane A, Riou J, Umbhauer M, Boucaut J, Shi D. 2000. Role of frizzled 7 in the regulation of convergent extension movements during gastrulation in Xenopus laevis. Development 127: 3091-3100.

Ema M, Takahashi S, Rossant J. 2006. Deletion of the selection cassette, but not cis-acting elements, in targeted Flk1-lacZ allele reveals Flk1 expression in multipotent mesodermal progenitors. Blood 107: 111-117.

Foley AC, Korol O, Timmer AM, Mercola M. 2007. Multiple functions of Cerberus cooperate to induce heart downstream of Nodal. Dev Biol 303: 57-65.

Fraidenraich D, Stillwell E, Romero E, Wilkes D, Manova K, Basson CT, Benezra R. 2004. Rescue of cardiac defects in id knockout embryos by injection of embryonic stem cells. Science 306: 247-252.

Gadue P, Huber TL, Paddison PJ, Keller GM. 2006. Wnt and TGF$\beta$ signaling are required for the induction of an in vitro model of primitive streak formation using embryonic stem cells. Proc Nat1 Acad Sci 103: 16806-16811.

Galvin KE, Travis ED, Yee D, Magnuson T, Vivian JL. 2010. Nodal signaling regulates the bone morphogenic protein pluripotency pathway in mouse embryonic stem cells. J Biol Chem 285: 19747-19756.

Hollnagel A, Oehlmann V, Heymer J, Ruther U, Nordheim A. 1999. Id genes are direct targets of bone morphogenetic protein induction in embryonic stem cells. I Biol Chem 274: 19838-19845.

Katagiri T, Imada M, Yanai T, Suda T, Takahashi N, Kamijo R. 2002. Identification of a BMP-responsive element in Id1, the gene for inhibition of myogenesis. Genes Cells 7: 949-960.

Kattman SJ, Huber TL, Keller GM. 2006. Multipotent flk- $1^{+}$cardiovascular progenitor cells give rise to the cardiomyocyte, endothelial, and vascular smooth muscle lineages. Dev Cell 11: 723-732.

Kee BL. 2009. E and ID proteins branch out. Nat Rev Immunol 9: 175-184.

Kee Y, Bronner-Fraser M. 2005. To proliferate or to die: role of Id3 in cell cycle progression and survival of neural crest progenitors. Genes Dev 19: 744-755.

Kelly RG, Brown NA, Buckingham ME. 2001. The arterial pole of the mouse heart forms from Fgf10-expressing cells in pharyngeal mesoderm. Dev Cell 1: 435-440. 
Kelly RG, Buckingham ME, Moorman AF. 2014. Heart fields and cardiac morphogenesis. Cold Spring Harb Perspect Med 4: a015750.

Korchynskyi O, ten Dijke P. 2002. Identification and functional characterization of distinct critically important bone morphogenetic protein-specific response elements in the Id 1 promoter. J Biol Chem 277: 4883-4891.

Laflamme MA, Chen KY, Naumova AV, Muskheli V, Fugate JA, Dupras SK, Reinecke H, Xu C, Hassanipour M, Police S, et al. 2007. Cardiomyocytes derived from human embryonic stem cells in pro-survival factors enhance function of infarcted rat hearts. Nat Biotechnol 25: 1015-1024.

Lescroart F, Chabab S, Lin X, Rulands S, Paulissen C, Rodolosse A, Auer H, Achouri Y, Dubois C, Bondue A, et al. 2014. Early lineage restriction in temporally distinct populations of Mesp1 progenitors during mammalian heart development. Nat Cell Biol 16: 829-840.

Lian X, Zhang J, Azarin SM, Zhu K, Hazeltine LB, Bao X, Hsiao C, Kamp TJ, Palecek SP. 2013. Directed cardiomyocyte differentiation from human pluripotent stem cells by modulating Wnt $/ \beta$-catenin signaling under fully defined conditions. Nat Protoc 8: 162-175.

Lopez-Rovira T, Chalaux E, Massague J, Rosa JL, Ventura F. 2002. Direct binding of Smad1 and Smad4 to two distinct motifs mediates bone morphogenetic protein-specific transcriptional activation of Id1 gene. J Biol Chem 277: 3176-3185.

Lyden D, Young AZ, Zagzag D, Yan W, Gerald W, O'Reilly R, Bader BL, Hynes RO, Zhuang Y, Manova K, et al. 1999. Id1 and Id 3 are required for neurogenesis, angiogenesis and vascularization of tumour xenografts. Nature 401: 670-677.

Marvin MJ, Di Rocco G, Gardiner A, Bush SM, Lassar AB. 2001. Inhibition of Wnt activity induces heart formation from posterior mesoderm. Genes Dev 15: 316-327.

McKeithan WL, Colas AR, Bushway PJ, Ray S, Mercola M. 2012. Serum-free generation of multipotent mesoderm $\left(\mathrm{Kdr}^{+}\right)$progenitor cells in mouse embryonic stem cells for functional genomics screening. Curr Protoc Stem Cell Biol 23: 1F.13.1-1F.13.13.

Meilhac SM, Esner M, Kelly RG, Nicolas JF, Buckingham ME. 2004. The clonal origin of myocardial cells in different regions of the embryonic mouse heart. Dev Cell 6: 685-698.

Meilhac SM, Lescroart F, Blanpain C, Buckingham ME. 2015. Cardiac cell lineages that form the heart. Cold Spring Harb Perspect Med 5: a026344.

Menasche P, Vanneaux V, Fabreguettes JR, Bel A, Tosca L, Garcia S, Bellamy V, Farouz Y, Pouly J, Damour O, et al. 2015. Towards a clinical use of human embryonic stem cell-derived cardiac progenitors: a translational experience. Eur Heart $I$ 36: 743-750.

Mercola M, Colas A, Willems E. 2013. Induced pluripotent stem cells in cardiovascular drug discovery. Circ Res 112: 534-548.

Miller EW, Lin JY, Frady EP, Steinbach PA, Kristan WB Jr, Tsien RY. 2012. Optically monitoring voltage in neurons by photoinduced electron transfer through molecular wires. Proc Natl Acad Sci 109: 2114-2119.

Moretti A, Laugwitz KL, Dorn T, Sinnecker D, Mummery C. 2013. Pluripotent stem cell models of human heart disease. Cold Spring Harb Perspect Med 3: a014027.

Nieuwkoop PD. 1967. The 'organization centre.' 3. Segregation and pattern formation in morphogenetic fields. Acta Biotheor 17: $178-194$.

Niola F, Zhao X, Singh D, Castano A, Sullivan R, Lauria M, Nam HS, Zhuang Y, Benezra R, Di Bernardo D, et al. 2012. Id proteins synchronize stemness and anchorage to the niche of neural stem cells. Nat Cell Biology 14: 477-487.
Niola F, Zhao X, Singh D, Sullivan R, Castano A, Verrico A, Zoppoli P, Friedmann-Morvinski D, Sulman E, Barrett L, et al. 2013. Mesenchymal high-grade glioma is maintained by the ID-RAP1 axis. J Clin Invest 123: 405-417.

Olson EN. 2006. Gene regulatory networks in the evolution and development of the heart. Science 313: 1922-1927.

Paige SL, Thomas S, Stoick-Cooper CL, Wang H, Maves L, Sandstrom R, Pabon L, Reinecke H, Pratt G, Keller G, et al. 2012. A temporal chromatin signature in human embryonic stem cells identifies regulators of cardiac development. Cell 151: 221-232.

Pandur P, Lasche M, Eisenberg LM, Kuhl M. 2002. Wnt-11 activation of a non-canonical Wnt signalling pathway is required for cardiogenesis. Nature 418: 636-641.

Peng HB. 1991. Xenopus laevis: practical uses in cell and molecular biology. Solutions and protocols. Methods Cell Biol 36: 657-662.

Roschger C, Cabrele C. 2017. The Id-protein family in developmental and cancer-associated pathways. Cell Commun Signal 15: 7.

Saga Y, Hata N, Kobayashi S, Magnuson T, Seldin MF, Taketo MM. 1996. MesP1: a novel basic helix-loop-helix protein expressed in the nascent mesodermal cells during mouse gastrulation. Development 122: 2769-2778.

Saga Y, Kitajima S, Miyagawa-Tomita S. 2000. Mesp1 expression is the earliest sign of cardiovascular development. Trends Cardiovasc Med 10: 345-352.

Schneider VA, Mercola M. 2001. Wnt antagonism initiates cardiogenesis in Xenopus laevis. Genes Dev 15: 304-315.

Schultheiss TM, Burch JB, Lassar AB. 1997. A role for bone morphogenetic proteins in the induction of cardiac myogenesis. Genes Dev 11: 451-462.

Stainier DY. 2002. A glimpse into the molecular entrails of endoderm formation. Genes Dev 16: 893-907.

Vincent SD, Dunn NR, Hayashi S, Norris DP, Robertson EJ. 2003. Cell fate decisions within the mouse organizer are governed by graded Nodal signals. Genes Dev 17: 1646-1662.

Viotti M, Nowotschin S, Hadjantonakis AK. 2014. SOX17 links gut endoderm morphogenesis and germ layer segregation. Nat Cell Biol 16: 1146-1156.

Wilkinson DG, Nieto MA. 1993. Detection of messenger RNA by in situ hybridization to tissue sections and whole mounts. Methods Enzymol 225: 361-373.

Wills AE, Baker JC. 2015. E2a is necessary for Smad2/3-dependent transcription and the direct repression of lefty during gastrulation. Dev Cell 32: 345-357.

Yang L, Soonpaa MH, Adler ED, Roepke TK, Kattman SJ, Kennedy M, Henckaerts E, Bonham K, Abbott GW, Linden RM, et al. 2008. Human cardiovascular progenitor cells develop from a $\mathrm{KDR}^{+}$embryonic-stem-cell-derived population. Nature 453: 524-528.

Yang J, Li X, Morrell NW. 2014. Id proteins in the vasculature: from molecular biology to cardiopulmonary medicine. Cardiovasc Res 104: 388-398.

Yoon SJ, Wills AE, Chuong E, Gupta R, Baker JC. 2011. HEB and E2A function as SMAD/FOXH1 cofactors. Genes Dev 25: 1654-1661.

Yoshida T, Vivatbutsiri P, Morriss-Kay G, Saga Y, Iseki S. 2008. Cell lineage in mammalian craniofacial mesenchyme. Mech Dev 125: 797-808.

Zhao R, Watt AJ, Battle MA, Li J, Bondow BJ, Duncan SA. 2008. Loss of both GATA4 and GATA6 blocks cardiac myocyte differentiation and results in acardia in mice. Dev Biol 317: 614-619. 


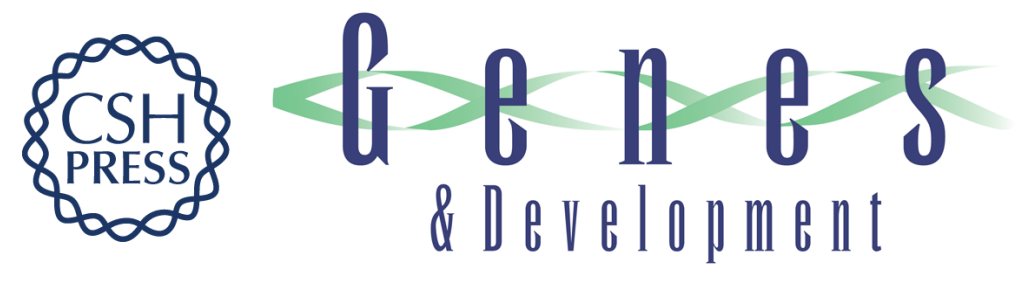

\section{Id genes are essential for early heart formation}

Thomas J. Cunningham, Michael S. Yu, Wesley L. McKeithan, et al.

Genes Dev. 2017, 31: originally published online August 9, 2017

Access the most recent version at doi:10.1101/gad.300400.117

\section{Supplemental \\ Material \\ http://genesdev.cshlp.org/content/suppl/2017/08/09/gad.300400.117.DC1 \\ References This article cites 66 articles, 25 of which can be accessed free at: \\ http://genesdev.cshlp.org/content/31/13/1325.full.html\#ref-list-1 \\ Creative This article, published in Genes \& Development, is available under a Creative Commons \\ Commons License (Attribution 4.0 International), as described at \\ License http://creativecommons.org/licenses/by/4.0/. \\ Email Alerting Receive free email alerts when new articles cite this article - sign up in the box at the top Service right corner of the article or click here.}

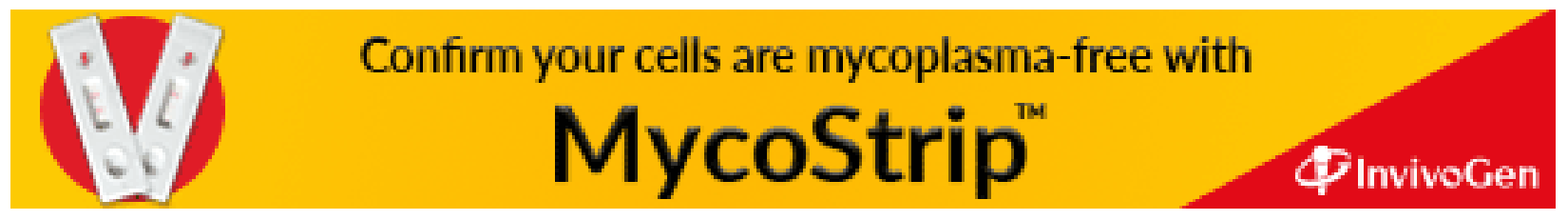

